\title{
VI. Laufende Forschungsprojekte zur Vertriebenenintegration
}




\section{Winfried Müller \\ Zum Forschungsverbund \\ „Die Entwicklung Bayerns durch die Integration der Heimatvertriebenen und Flüchtlinge“}

Ungeachtet einiger zentraler Publikationen zur Flüchtlingspolitik und -integration im Bayern der Nachkriegszeit ${ }^{1}$, blieb - gerade weil die Forschungslage auch von Phasen der Stagnation gekennzeichnet war - die Dringlichkeit einer wissenschaftlichen Aufbereitung der Aufnahme und Eingliederung der Vertriebenen und Flüchtlinge in Wissenschaft und Politik unbestritten. Für letztere gilt dabei der klare Auftrag des $\$ 96$ des Bundesvertriebenengesetzes an Bund und Länder, „Wissenschaft und Forschung bei der Erfüllung der Aufgaben, die sich aus der Vertreibung und der Eingliederung der Vertriebenen und Flüchtlinge ergeben“, „zu fördern“. Im Bewußtsein dieser Verpflichtung legte das Bayerische Sozialministerium bereits 1984 die von ihm in Auftrag gegebene zweibändige Dokumentation „Integration und Neubeginn“ vor, die die Eingliederung der Wirtschaftsbetriebe der Vertriebenen in Bayern zum Inhalt hat ${ }^{2}$. Zu verweisen ist ferner auf die 1989 gemeinsam von dem Sozialministerium, der Forschungsgemeinschaft für das Weltflüchtlingsproblem und dem Sudetendeutschen Archiv herausgegebene kommentierte Bibliographie zum Flüchtlings- und Vertriebenenproblem³. Gleichfalls 1989 begonnen und 1991 abgeschlossen wurde die von der Sudetendeutschen Akademie der Wissenschaften und Künste in Auftrag gegebene und vom Sozialministerium geförderte Untersuchung der Integration vertriebener Behinderter in der Bundesrepublik Deutschland unter besonderer Berücksichtigung des Freistaates Bayern ${ }^{4}$.

Das längerfristig wohl wichtigste und umfangreichste Projekt des Bayerischen Staatsministeriums für Arbeit und Sozialordnung, Familie, Frauen und Gesundheit im Bereich der Wissenschaftsförderung ist aber der hier im Mittelpunkt stehende Forschungsverbund „Die Entwicklung Bayerns durch die Integration der Vertriebenen und Flüchtlinge $^{\text {“5 }}$. Erste Überlegungen hierzu gehen auf das Jahr 1985 zurück, als sich der Beginn von Flucht und Vertreibung zum 40. Mal jährte. Die endgültige Entscheidung über das Projekt fiel 1989, sein Start erfolgte 1990. Koordinationszentrum wurde Bayreuth, wo

1 Vgl. u. a. Kornrumpf, In Bayern angekommen; Bauer, Flüchtlinge und Flüchtlingspolitik. Zusammenfassung des Forschungsstandes bei Lanzinner, S. 91.

2 Vgl. Prinz, Integration und Neubeginn.

${ }^{3}$ Vgl. Krallert-Sattler, Kommentierte Bibliographie.

+ Vgl. Baier/Lamprecht, Integration.

${ }^{5}$ Als außerbayerisches Pendant hierzu vgl. das an der Universität Mannheim angesiedelte, von Wolfgang von Hippel betreute und von der Stiftung Volkswagenwerk sowie vom Ministerium für Wissenschaft und Forschung des Landes Baden-Württemberg finanziell geförderte Forschungsprojekt „Die Integration der Flüchtlinge und Heimatvertriebenen in Baden und Württemberg nach $1945^{\circ}$. Erste Teilergebnisse bei Schraut. Vgl. auch Beer, Flüchtlinge; Beer, Integration. 
im Zusammenspiel mit Lastenausgleichsarchiv und Universität der Flüchtlings- und Eingliederungsforschung eine stetig wachsende Bedeutung zukommen dürfte. Die Projektkoordination liegt beim langjährigen Präsidenten der Universität Bayreuth und Gründungsbeauftragten der Universität Erfurt, Klaus Dieter Wolff, und - für die federführende Behörde - bei Ministerialrat Walter Rösner-Kraus.

Das Projekt ist zum einen interdisziplinär ausgerichtet, zum anderen - im Begriff des Forschungsverbunds kommt dies zum Ausdruck - ist es universitätsübergreifend auf die Einbeziehung aller Hochschulen des Landes Bayern angelegt. Das Projekt verfolgt weniger eine deskriptive und statistische Aufarbeitung der Aufnahme und Eingliederung der Vertriebenen und Flüchtlinge. Vielmehr will es unter Zugrundelegung der Forschungsprämisse "Wandel durch Integration"6 primär der Frage nachgehen, welche Auswirkungen der Zustrom von mehr als zwei Millionen Vertriebenen und Flüchtlingen auf Bayerns Wirtschaft und Gesellschaft, auf Politik und Verwaltung, auf Kultur- und Geistesleben hatte. Zentrale Fragestellung ist somit: welche Impulse gingen nach dem Neubeginn des Jahres 1945 von den Heimatvertriebenen und Flüchtlingen für die Modernisierung des Aufnahmelandes Bayern aus. Erwartet werden ebenso Erkenntnisse über Integrationswege und -voraussetzungen, integrationsfördernde Entscheidungen und Maßnahmen sowie integrationshemmende Faktoren.

Für die Publikation der einschlägigen Untersuchungen wurde eine eigene, seit 1993 erscheinende Reihe eingerichtet. Sie wird herausgegeben von Hermann-Joseph Busley, Rudolf Endres, Otto Kimminich, Jörg Maier und Klaus Dieter Wolff. Komprimierte Ergebnisauszüge der dort aufgenommenen Studien präsentiert eine aus Anlaß des Gedenkjahres 1995 herausgegebene Sonderpublikation?

Innerhalb der Reihe wurde zunächst 1993 eine Studie von Otto Kimminich (Universität Regensburg) publiziert ${ }^{8}$, die in der Systematik des Gesamtvorhabens insofern einen Ausgangspunkt darstellt, als sie den völkerrechtlichen Hintergrund der Aufnahme und Integration der Heimatvertriebenen und Flüchtlinge in Bayern thematisiert. Es geht dabei nicht nur um die Rechtslage im besetzten Deutschland und um Statusfragen der Vertriebenen und Flüchtlinge, sondern ebenso um die staats- und völkerrechtliche Entwicklung in den Vertreibungsräumen seit den zwanziger Jahren unter Einschluß der Nationalitätenkonflikte, Kriegsereignisse, Kriegsziele, Umsiedlungspläne und Gesetzeswerke der die Vertreibung durchführenden Staaten.

Abgeschlossen ist ferner das von Johann Handl und Christa Herrmann an der Universität Erlangen durchgeführte Teilprojekt „Soziale und berufliche Umschichtung der Bevölkerung in Bayern nach 1945"9. Diese Analyse der Mikrozensus-Zusatzerhebung von 1971 vergleicht die beruflichen Entwicklungslinien von Vertriebenen, SBZ-Flüchtlingen und Einheimischen und thematisiert in einem besonderen Teil die Heiratsbeziehungen zwischen diesen Bevölkerungsgruppen. Korrespondierend dazu befaßt sich die vor dem Abschluß stehende, gleichfalls an der Universität Erlangen angesiedelte Untersuchung „Lebens- und Biographieverläufe von Vertriebenen und Flüchtlingen“ von Michael von

${ }^{6}$ Wolff, Wandel durch Integration, S. 21.

7 Vgl. Bayerisches Staatsministerium für Arbeit und Sozialordnung, Familie, Frauen und Gesundheit (Hrsg.), Die Entwicklung Bayerns.

${ }^{8}$ Vgl. Kimminich, Völkerrechtlicher Hintergrund. Vgl. zusammenfassend auch Kimminich, Flucht und Vertreibung, S. 17-34.

9 Vgl. Handl/Herrmann, Soziale und berufliche Unterschiede; zusammenfassend ferner Handl, Sozialstrukturelle Aspekte, S. 35-47. 
Engelhardt mit der Erfahrungs- und Bewältigungsgeschichte der Neubürger ${ }^{10}$. Was im Teilprojekt von Handl/Herrmann auf der Makroebene ermittelt wurde, soll hier auf der Mikroebene durch Einzelfalluntersuchungen ergänzt werden.

Nachdem zwischenzeitlich die aus Drittmitteln finanzierte Dissertation von Gerald Neumann über die Behandlung der Flüchtlingsfrage in den Medien in die Forschungsreihe ${ }^{11}$ aufgenommen worden war, erschien zuletzt schließlich die wiederum auf einem Teilprojekt beruhende Untersuchung von Jörg Maier und Germano Tullio (Universität Bayreuth), die sich mit der sozialen und wirtschaftlichen Eingliederung von Flüchtlingen und Heimatvertriebenen in Bayern befaßt. Aufbauend auf einer Darstellung von Integrationswiderständen und Integrationsstufen wird dabei an ausgewählten Untersuchungsgebieten den Konsequenzen des Bevölkerungszustroms - es waren ja nicht weniger als über 400000 Erwerbspersonen aufzunehmen - für den wirtschaftsstrukturellen Wandel in Bayern nach dem Zweiten Weltkrieg nachgegangen. Vor allem - so ein Ergebnis dieser Studie - das verarbeitende Gewerbe (Textil und Bekleidung, chemische Industrie, Feinkeramik, Glasindustrie, Ledererzeugung und -verarbeitung) ermöglichte eine Beteiligung der Neubürger. „So erhielt die bayerische Industrie einen qualifizierten Zugang gerade in der verarbeitenden Industrie, der die Volkswirtschaft des rohstoffarmen, aber nun arbeitskräftestarken Landes nachhaltig stärken sollte"12.

Unmittelbar vor dem Abschluß steht das von Walter Ziegler ${ }^{13}$ betreute Teilprojekt „Die Vertriebenen vor der Vertreibung. Die Heimatländer der deutschen Vertriebenen im 19. und 20. Jahrhundert: Strukturen, Entwicklungen, Erfahrung", das in der Systematik des Gesamtprojekts neben der bereits erwähnten Studie von Otto Kimminich den Ausgangspunkt bilden wird. Ausgangsüberlegung dieses Vorhabens ist das Faktum, daß das Interesse an der Geschichte der Vertriebenen und Flüchtlinge sich primär auf die Gründe und den Vorgang der Vertreibung einerseits, auf die Aufnahme und Integration in der neuen Heimat andererseits konzentrierte. Das bedeutete, daß die Frage nach dem konkreten Umfeld, nach dem politischen und kulturellen Milieu, dem die Heimatvertriebenen und Flüchtlinge entwurzelt wurden, in den Hintergrund trat. Indem deren Geschichte, die ja auch für die Integration im Aufnahmeland ein bestimmender Faktor war und ist, in ihrem Facettenreichtum aufgearbeitet wird, soll zugleich deutlich werden, wie verkürzend im Grunde die begriffliche Reduzierung auf „die Deutschen“ bzw. „die Flüchtlinge“ ist. „Nicht zuerst, die Deutschen' flüchteten und wurden vertrieben, sondern Bürger aus Eger oder Forstarbeiter aus dem Böhmerwald, Bergleute aus der Unterzips, Bauern aus Bessarabien, aber auch Fischer aus Pommern oder Großgrundbesitzer aus Ostpreußen. Und verlassen mußten sie nicht einfach ,Ostgebiete““, sondern „Heimatgebiete verschiedenster Art und unterschiedlichster Befindlichkeit"14. Die Geschichte und Kulturgeschichte dieser Gebiete in ihrer Gesamtheit von der Mitte des 19. Jahrhunderts bis zum Zeitpunkt von Flucht und Vertreibung handbuchartig aufzubereiten ist das eine Anliegen des von Ziegler betreuten Projektes. Ein zweites ist es dann, auf der Basis dieser Einzeldar-

10 Vgl. Engelhardt, Biographieverläufe, S. 49-77.

11 Vgl. auch den zusammenfassenden Bericht von Neumann, Medien, S. 93-101.

12 Maier, S. 92. Vgl. vor allem Maier/Tullio, Die soziale und wirtschaftliche Eingliederung. Als ergänzende Spezialstudie vgl. auch Fruhmann, Geographische Untersuchungen, S. 1-201.

13 Prof. Dr. Walter Ziegler, Institut für Bayerische Geschichte der Ludwig-Maximilians-Universität München, Ludwigstr. 14, 80539 München.

14 Ziegler, Die verlorene Heimat, S. 105. Bei dem hier zitierten Beitrag handelt es sich um einen Vorbericht zum skizzierten Teilprojekt. 
stellungen Vergleichsmöglichkeiten zu eröffnen. Als mögliche Fragestellung bieten sich beispielsweise an: die Stellung der Deutschen in den jeweiligen Nationalstaaten, in denen sie nach 1918 beheimatet waren, ihr Verhältnis zur NS-Diktatur, die jeweilige Situation unmittelbar vor der Vertreibung. Schließlich, und dies ist das dritte Anliegen, soll das Teilprojekt „Die Vertriebenen vor der Vertreibung“ helfen, „die Verkrustungen des Nationalismus zu lösen und die gemeinsame Geschichte, freilich auch die gemeinsam verschuldeten Probleme dieser Geschichte zu erfassen und das Terrain für künftiges friedliches Miteinander neu zu bereiten "15.

$\mathrm{Zu}$ den bislang skizzierten, bereits abgeschlossenen Teilprojekten gesellten sich 1995 zwei weitere Arbeitsvorhaben, die kurz vor dem Abschluß stehen; mit der Publikation der Ergebnisse dürfte demnach 1999 zu rechnen sein. Das eine der beiden neuen Teilprojekte ist am Institut für deutsche und vergleichende Volkskunde der Universität München angesiedelt. Es wird von Helge Gerndt betreut ${ }^{16}$ und steht unter dem Titel „Vereinswesen als Integrationsfaktor. Eine volkskundliche Studie zum Wandel des Vereinswesens in Bayern durch den Zuzug der Heimatvertriebenen und Flüchtlinge nach 1945“. Von der Prämisse ausgehend, daß die Erörterung der Integration der Heimatvertriebenen und Flüchtlinge in der Literatur häufig auf die Ebene der wirtschaftlichen und sozialen Integration beschränkt bleibt, wohingegen unsere Kenntnis über die Integration im Alltagsleben und über private Verkehrskreise relativ gering ist, will dieses Teilprojekt der Frage nachgehen, wieweit Vereine als Orte der Begegnung Aufschluß geben über konkrete Wege der kulturellen Eingliederung von Heimatvertriebenen und Flüchtlingen in Bayern. Das Forschungsvorhaben will eine erste Bestandsaufnahme der Veränderungen des Vereinswesens durch den Zuzug der Vertriebenen und Flüchtlinge leisten. Ziel ist es, die kulturellen Annäherungsprozesse von Aufnahmegesellschaft und „Neubürgern" anhand des Vereinslebens zu beschreiben und vergleichend zu analysieren. Das Erkenntnisinteresse gilt den Einflüssen, die die kulturellen Verhaltensweisen und Anschauungen auf den Integrationsprozeß ausüben. Da das Vereinswesen für die Zeit nach 1945 staatlicherseits statistisch nicht erfaßt ist, gilt es, in einem ersten Arbeitsschritt für ausgewählte bayerische Landkreise eine Datenbasis zu schaffen, die Veränderungen in der Vereinsstruktur in der Nachkriegszeit aufzeigbar macht. Hierzu müssen die bei den Amtsgerichten geführten Vereinsregister gesichtet und ausgewertet werden. Auf der Grundlage bereits veröffentlichter Daten (Siedlungsstruktur, Sozialstruktur etc.) zur Entwicklung Bayerns durch die Eingliederung der Vertriebenen und Flüchtlinge sowie der gewonnenen Daten aus der ersten Projektphase ist eine qualitative Mikrostudie des Vereinswesens in einer oder mehreren Gemeinden geplant. Neben der Durchsicht und Auswertung eventuell vorhandener schriftlicher und bildlicher Quellen (Protokollbücher, Vereinschroniken etc.) sind insbesondere lebensgeschichtliche Interviews mit Vereinsmitgliedern geplant.

Das zweite 1995 gestartete Teilprojekt widmet sich dem Integrationsprozeß im Bildungssektor und wird vom Verfasser des vorliegenden Berichts betreut ${ }^{17}$. Die Darstel-

15 Ebenda, S. 103.

${ }^{16}$ Prof. Dr. Helge Gerndt, Institut für deutsche und vergleichende Volkskunde, Ludwig-Maximilians-Universität München, Ludwigstr. 25, 80539 München. Anfragen sind zu richten an die für das Teilprojekt zuständige Mitarbeiterin Frau Astrid Pellengahr M. A., der ich für die nachfolgend mitgeteilte Projektbeschreibung danke.

17 Prof. Dr. Winfried Müller, z.Z. Univ. Bonn, c/o Mittelalterliche und vergleichende Landesgeschichte am Institut für Bayerische Geschichte der Ludwig-Maximilians-Universität München, Schellingstr. 5, 80799 München. 
lung der Integration der Vertriebenen und Flüchtlinge im Bildungssektor und der daraus resultierenden Impulse für die Entwicklung des Aufnahmelandes ist nicht nur im Rahmen des bayerischen Forschungsverbundes ein Desiderat: Die Bildungseinrichtungen, in erster Linie die Schulen, waren die Institutionen, in denen sich die Kinder und Jugendliche der Einheimischen und Neubürger zunächst am intensivsten begegneten, wo es zu einer wechselseitigen Annäherung und osmotischen Durchdringung jeweils autochthoner Traditionen und konfessioneller Milieus kam. Sie waren die Stätten, in denen die junge Generation unter den Vertriebenen und Flüchtlingen ihre berufsqualifizierenden Abschlüsse anstrebte, die Voraussetzung für die soziale und wirtschaftliche Integration nach dem Zweiten Weltkrieg waren; den Aufnahmeländern kam hier ein enormes Bildungspotential zugute. Für die Erwachsenengeneration unter den Vertriebenen und Flüchtlingen war, gerade weil ihnen oft nur das "geistige Fluchtgepäck" geblieben war, der Bildungssektor ein attraktives Betätigungsfeld: die Bildungseinrichtungen waren es, die den Vertriebenen und Flüchtlingen teilweise ungewöhnlich gute Chancen zur beruflichen Eingliederung boten. So wurde ihnen ermöglicht, ihre Qualifikationen und Erfahrungen einzubringen und für das Aufnahmeland fruchtbar werden zu lassen. Es erstaunt deshalb, daß der Integrationsprozeß im Bildungssektor bislang weder für Bayern noch für die anderen Bundesländer hinlänglich beachtet wurde.

Für den Zugang zum Thema bieten sich drei Ebenen an, für die nachfolgend einige auf Bayern bezogene Daten mitgeteilt werden sollen: das allgemeinbildende Schulwesen, die Universitäten und Hochschulen und schließlich die Erwachsenenbildung. Was die Schulen betrifft, so drängt sich als ein wichtiger Teilaspekt zunächst die Integration und Tätigkeit der "Flüchtlingslehrer" in Bayern auf. Wie bereits Franz J. Bauer in seiner den Bildungsbereich nur am Rande streifenden Untersuchung über „Flüchtlinge und Flüchtlingspolitik in Bayern 1945-1950“ (1982) feststellte, gelang es den „Lehrern unter den Flüchtlingen mehr als den Angehörigen irgendeiner anderen qualifizierten Berufsgrup$\mathrm{pe}^{\text {"18 }}$ eine ihrer Ausbildung und ihrem früheren Status adäquate Beschäftigung zu finden. Dabei spielten mehrere Faktoren zusammen: der durch Kriegsverluste, Entnazifizierung und den sprunghaften Anstieg der Schülerzahlen verursachte Lehrermangel der Nachkriegszeit, die hoch zu veranschlagende Integrationswilligkeit der bayerischen Unterrichtsverwaltung, die ca. $20 \%$ der Planstellen für die Vertriebenen und Flüchtlinge vorsah (diese Quote wurde teilweise erheblich überschritten), nicht zuletzt aber auch Initiative und Engagement der "Flüchtlingslehrer". In diesem Zusammenhang dürfte es von Interesse sein, der Arbeit der „Zentralstelle für Flüchtlingslehrer" nachzugehen, die 1945 zunächst in der britischen Zone ihren Ausgang genommen hatte, später dann von den deutschen Ländern - unter Einschluß Bayerns - getragen wurde, ehe sie 1952 in einer Abteilung des Bundesinnenministeriums aufging. Die erfolgreiche Vermittlungstätigkeit der Zentralstelle, bei der sich ca. 60000 „Flüchtlingslehrer" registrieren ließen, ist so gut wie unerforscht ${ }^{19}$.

Das hohe Maß der beruflichen Integration der „Flüchtlingslehrer“ und der damit verbundene Wissenstransfer machten die Schule zweifelsohne zu einem wichtigen Ort der Interaktion von Einheimischen und Neubürgern. Das Ausmaß der mentalen und kulturellen Prägung Bayerns durch die „Flüchtlingslehrer" mag sich letztlich gesicherter Eva-

${ }^{18}$ Bauer, Flüchtlinge, S. 209. Zur Integration der „Flüchtlingslehrer“ vgl. auch die Hinweise bei Müller, Schulpolitik, S. $83 \mathrm{ff}$.

${ }^{19}$ Vgl. Müller, Schulpolitik, S. 85 f. 
luierung entziehen. Gerade deshalb bietet sich ergänzend als zweites wichtiges Arbeitsfeld die Schulbuch- und Lehrplananalyse an, wobei im Hinblick auf die Behandlung des Themas Flucht und Vertreibung sowie der Ostkunde vor allem die Fächer Heimatkunde, Geschichte und Geographie, eventuell auch der Religionsunterricht, besondere Berücksichtigung finden müssen. Zunächst sind hier die amtlichen Richtlinien für den Unterricht an Bayerns Schulen eingehend zu überprüfen; in erster Linie Lehrpläne der diversen Schularten, des weiteren Modelle für den Unterricht, sicherlich aber auch Vorstellungen und Konzepte engagierter Lehrerinnen und Lehrer. Als Beispiele seien nur genannt die Bekanntmachungen des Kultusministeriums zur Behandlung der Ostkunde im Unterricht, die Tagungsberichte der "Akademie für Lehrerfortbildung“ in Dillingen oder die Ergebnisse der "Arbeitsgemeinschaft sudetendeutscher Erzieher". Organisationen wie etwa der Verband der Geschichtslehrer Deutschlands sind auf entsprechende Maßnahmen zu untersuchen. Daß eine differenzierte Sichtung der Unterrichtsmaterialien, insbesondere der Schulbücher, aber auch anderer Medien (Schulfilm, Schulfunk), zu leisten ist, versteht sich von selbst. Die Vielfalt der von den jeweiligen Landsmannschaften publizierten Organe, ob nun Zeitungen oder wissenschaftliche Zeitschriften, bietet ein weiteres Aufgabenfeld.

Dritter wichtiger Teilaspekt für den Bereich des allgemeinbildenden Schulwesens ist die Integration und Ausbildung der Kinder und Jugendlichen unter den Vertriebenen und Flüchtlingen ${ }^{20}$. Es gilt zu bedenken, daß sich beispielsweise bereits im Oktober 1946163963 Flüchtlingskinder unter sechs Jahren in Bayern aufhielten, deren Einschulung bevorstand, 238289 Flüchtlingskinder im volksschulpflichtigen Alter, ferner 163428 Jugendliche zwischen 14 und 20 Jahren, die zumindest teilweise in die Berufsschulen, höheren Schulen und Universitäten strebten. In diesem Zusammenhang bietet es sich an, Erkenntnisse über Bildungsverhalten und Schulabschlüsse zu gewinnen. Gab es etwa im Sinne einer besonderen Motivation zum Bildungserwerb in einer Altersgruppe bzw. Schülerjahrgängen signifikante Abweichungen des Anteils der Vertriebenen und Flüchtlinge an den Realschülern und Abiturienten in Relation zum Anteil dieser Bevölkerungsgruppe an der Gesamtbevölkerung? In Weiterung dessen müßte dann - wiederum unter der dem Gesamtprojekt zugrundeliegenden Fragestellung des Beitrags zur Entwicklung Bayerns - der berufliche Werdegang jener aufgeschlüsselt werden, die nach Flucht und Vertreibung ihren Schulabschluß in Bayern erwarben. Aufgrund der bislang eingesehenen Unterlagen des Bayerischen Statistischen Landesamtes scheint es möglich, hier zu aussagekräftigen Ergebnissen zu kommen. Daneben sollten aber auch exemplarisch individuelle Karriereverläufe geschildert werden. Schließlich ist - vor allem im Hinblick auf die Volksschule, wo man nach 1945 zu der vom NS-Regime beseitigten Konfessionsschule zurückkehrte - als gesondertes Themenfeld noch der konfessionelle Aspekt auszuweisen ${ }^{21}$. Mit dem Zustrom der Vertriebenen und Flüchtlinge gehörte die konfessionelle Homogenität Bayerns auf der mikrostrukturellen Ebene der Vergangenheit an. Während sich auf der Makroebene die Proportionen zwischen den Konfessionen kaum verschoben, da die Konfessionsstruktur der Neubürger weitgehend mit der des Aufnahmelandes Bayern korrespondierte, verschwanden auf der lokalen Ebene konfessionell homogene Gemeinden fast vollständig; 1939 hatte es in Bayern noch 1352 ausschließlich katholische Gemeinden gegeben, 1953 waren es noch 27, d. h., daß der Akkulturations-

20 Vgl. ebenda, S. $62 \mathrm{ff}$.

21 Vgl. ebenda, S. $67 \mathrm{ff}$. Vgl. auch Erker, Die Revolution des Dorfes, S. $382 \mathrm{ff}$. 
prozeß auf der lokalen Ebene, das Nebeneinander von Einheimischen und Neubürgern, die jeweils ihre eigene Tradition einbrachten und an ihrer Identität festhalten wollten, auch starke Veränderungen im konfessionellen Milieu implizierte. Daß das - gerade im Zuge der Rekonfessionalisierung des Volksschulwesens - auf beiden Seiten auch mit Reibungsverlusten verbunden war, braucht nicht beschönigt werden. Gleichzeitig gilt es aber, der Rolle der Schule als Ort der Begegnung und Verschmelzung unterschiedlicher landsmannschaftlicher und konfessioneller Milieus nachzugehen. Hier läßt eine Auswertung der Schulberichte der Bezirksregierungen, die in den Akten des Bayerischen Staatsministeriums für Unterricht und Kultus, Wissenschaft und Kunst vorliegen, ferner der Berichte der Dienststellen der amerikanischen Militärregierung gewichtige Aufschlüsse erwarten.

Was den Hochschulbereich als das zweite zentrale Untersuchungsfeld im Rahmen des Teilprojekts „Bildungswesen“ betrifft, so müßte hier zunächst einmal, in Analogie zu den „Flüchtlingslehrern“, die Frage nach der Eingliederung der vertriebenen und geflüchteten Hochschullehrer aufgegriffen werden. Hier taten sich, anders als im Schulbereich, offenkundig enorme Schwierigkeiten auf. Denn während das Kultusministerium für die Schulen eine Quote festlegen konnte, waren ihm im Hochschulbereich weitgehend die Hände gebunden. Dort wurden nämlich freie Wissenschaftlerstellen aufgrund der traditionellen, nach 1945 wiederhergestellten akademischen Selbstverwaltung auf Vorschlag der Fakultäten besetzt. Dieses dezentralisierte Verfahren erwies sich für die berufliche Eingliederung gerade der älteren unter den „Flüchtlingsprofessoren“ nicht unbedingt förderlich. Selbsthilfeorganisationen der Flüchtlinge und Vertriebenen vertraten deshalb gegenüber dem Ministerium mit Eingaben und Petitionen wiederholt die in bedrängter Situation lebenden, zum Teil immerhin mit Lehraufträgen ausgestatteten Professoren, wobei u. a. auch die Errichtung sog. Flüchtlings-Notprofessuren angeregt wurde. Letztere Bemühungen wurden zu Beginn der fünfziger Jahre durch das Ausführungsgesetz zu Artikel 131 des Grundgesetzes auf eine neue Grundlage gestellt, das seitens des bayerischen Kultusministeriums gezielt für die Schaffung von kw (künftig wegfallend)Stellen für vertriebene und geflüchtete Professoren genützt wurde ${ }^{22}$. Der Blick ist freilich nicht nur auf unverkennbare Eingliederungsschwierigkeiten zu richten. Zugleich ist zu betonen, daß sich mit den durch Flucht und Vertreibung nach Bayern gelangten Hochschullehrern - sei es, daß sie aus Prag, Breslau, Königsberg, Posen und Danzig oder von den Hochschulen der sowjetisch besetzten Zone wie Jena, Leipzig oder Dresden kamen - nicht nur die Chance bot, personelle Lücken zu schließen, sondern auch hervorragende Gelehrte zu gewinnen, die der Wissenschaftsentwicklung nach $1945 \mathrm{z}$. T. wichtige Impulse gaben. So wurden, um nur einige wenige Namen zu nennen, beispielsweise die Kirchenhistoriker Berthold Altaner und Franz Xaver Seppelt, beide vormals in Breslau tätig, nach Würzburg und München berufen. Der Dresdener Psychologe Gustav Kafka erhielt einen Ruf nach Würzburg. Gerhard Eis, der mit der Erforschung der mittelalterlichen Fachprosa seiner Disziplin neue Impulse gab, fand, aus Prag kommend, zunächst in Passau eine neue Wirkungsstätte.

Dieser Problemkreis der Integration im Wissenschaftssektor ist noch nicht einmal ansatzweise erforscht, obwohl die Quellenlage durchaus gut ist, wie u. a. jüngst abgegebene Akten des bayerischen Kultusministeriums erweisen. Dabei zeigt es sich im übrigen, daß

${ }^{22}$ Vgl. Müller, Universitäten. Vgl. auch Günther, Die Unterbringung, S. $126 \mathrm{ff}$.; Wendehorst, Gcschichte, S. 240. 
nicht nur die Universitäten Erlangen, München und Würzburg zu beachten sind, sondern auch die in Bayern bis zur Universitätsgründungswelle der späten sechziger Jahre existierenden Philosophisch-Theologischen Hochschulen. Es scheint, daß an ihnen vielfach Gelehrte aus dem Kreis der Flüchtlinge und Vertriebenen zumindest vorübergehend eine neue Wirkungsstätte fanden. So wurde in Regensburg eine ganze Reihe von Medizinern aufgenommen, die Prag hatten verlassen müssen. In Passau lehrte, wie oben erwähnt, für einige Zeit Gerhard Eis.

Für den Hochschulbereich bietet sich ferner die Frage nach den Zulassungsbedingungen und dem Anteil der Vertriebenen und Flüchtlinge unter den Studenten an, den von ihnen bevorzugten Fachrichtungen und gewählten Berufen. Zudem wird nachzufragen sein, welche Initiativen für die wissenschaftliche Erforschung der Geschichte und Kultur der Ostdeutschen bzw. der in Osteuropa lebenden Deutschen nach 1945 im Hochschulbereich eingebracht wurden, etwa durch Lehrstuhlgründungen, und welche Ergebnisse sie zeitigten.

Dritte Untersuchungsebene im Bereich des Bildungssektors sollte schließlich die Erwachsenenbildung sein. Im Rahmen zusätzlichen Qualifikationserwerbs, aber auch hinsichtlich der Pflege der kulturellen Überlieferung, dem Praktizieren festgefügter Gemeinsamkeiten während der schwierigen Anfangsjahre in der zweiten Heimat kam ihren Angeboten besondere Bedeutung zu. Hier ist zunächst an die Eigeninitiativen der Vertriebenen und Flüchtlinge - Tagungen und Publikationstätigkeit von Verbänden und Gruppen - zu denken; die Arbeit der Stiftung Kulturwerk Schlesien in Würzburg mag als eindrucksvolles Beispiel stehen. Gleichzeitig sollte der Frage nachgegangen werden, inwieweit die Interessen der Vertriebenen und Flüchtlinge beim Wiederaufbau des Volkshochschulwesens berücksichtigt wurden und wie diese selbst bei der programmatischen Ausgestaltung der Volkshochschulen initiativ wurden. Eine neuere Untersuchung ${ }^{23}$ zeigt, daß die integrierende Funktion der Volkshochschulen sehr hoch anzusetzen ist und daß deren regionale Streuung nach 1945 nicht zuletzt deshalb erfolgte, um die Flüchtlinge und Vertriebenen in den ländlichen Gebieten zu erreichen. Die Gründung einer Arbeitsgemeinschaft des „Bayerischen Landesverbandes für freie Volksbildung“, des „Hauptausschusses für Flüchtlinge und Ausgewiesene“ und des „Kulturellen Arbeitskreises der deutschen Heimatverwiesenen" verfolgte das erklärte Ziel, die Volkshochschulen als Begegnungsstätten zwischen Vertriebenen und Einheimischen zu etablieren. Auf der einen Seite wurden so vermehrt Themen über und für Vertriebene und Flüchtlinge angeboten und Aufklärungsarbeit über deren Kultur und Probleme geleistet, auf der anderen Seite sollte den Vertriebenen und Flüchtlingen Gelegenheit gegeben werden, das „Kulturgut der neuen Heimat" kennenzulernen. Für die Akzeptanz dieses Vorhabens spricht es, daß 1950 ca. $32 \%$ der Hörer und ca. 38\% der Dozenten an Volkshochschulen aus dem Kreis der Vertriebenen und Flüchtlinge stammten. So gesehen kommt der Erörterung der bislang kaum beachteten Volkshochschulen in unserem Problemzusammenhang hoher Rang zu.

Anzusprechen wird dabei auch das von der amerikanischen Militärregierung mitinitiierte und finanzierte Grenzlandprogramm sein, in dessen Verlauf Vertreter der Volkshochschulen in die strukturschwachen Grenzgebiete in kultureller Rand- und politischer Gefahrenlage reisten und in beachtlichem Umfang Einheimische und Vertriebene gleichermaßen in die Erwachsenenbildung einbezogen - auch in der Absicht, kommunisti-

${ }^{23}$ Vgl. Hummel, Wiederaufbau. 
scher Propaganda entgegenzuwirken. Gerade das Beispiel der Grenzlandfahrten legt im übrigen die - generell für alle bislang berührten Aspekte geltende - Frage nach regionalen Spezifizierungen nahe. Mögliches Untersuchungskriterium wäre, ob sich in grenznahen Siedlungen eine verstärkte Auseinandersetzung mit dem Thema Flucht und Vertreibung beobachten läßt bzw. ob sich dort spezielle Ausformungen eines durch die Vertriebenen und Flüchtlinge geförderten politischen und kulturellen Bewußtseins entwickelt haben.

Zu diesen zuletzt skizzierten, 1995 begonnenen Teilprojekten soll die Erschließung weiterer Forschungsfelder hinzutreten. So sind im Rahmen des Gesamtprojekts beispielsweise Studien denkbar zum Wandel der Frömmigkeitsformen, zu Entwicklungen des Brauchtums und Veränderungen der Alltagskultur, zur Rolle der Kirchen und karitativen Organisationen bei der Aufnahme und Eingliederung der Neubürger, zur Partizipation der Vertriebenen am politischen Leben u. a.m. Am Ende sämtlicher Einzeluntersuchungen wird dann ein eigener Auftrag mit dem Ziel vergeben werden, ein Fazit mit aktuellen Schlußfolgerungen aus allen Teilprojekten zu ziehen. Insgesamt soll dann eine Forschungsreihe vorliegen, die umfassend die Impulse dokumentiert, die durch die Aufnahme der Vertriebenen und Flüchtlinge zum Umbau Bayerns nach dem Krieg beigetragen haben.

Die zuletzt avisierte Einbeziehung weiterer Teilaspekte und die angestrebte Abrundung des Gesamtprojekts dürften durch zwei Faktoren erleichtert werden: Zum einen ist seit der Initiierung des Projekts eine deutliche Zunahme von Seminar- und Abschlußarbeiten einschlägiger Thematik an den bayerischen Universitäten zu beobachten, d. h., es wurde eine Förderung wissenschaftlichen Nachwuchses auf dem Feld der Eingliederungsforschung bewirkt. Zum anderen wurde unter der Leitung von Rudolf Endres an der Universität Bayreuth eine Dokumentationsstelle eingerichtet, die seit August 1990 der Ermittlung bisher nicht erfaßter Quellen in staatlichen, kommunalen, kirchlichen, verbandseigenen und privaten Archiven Bayerns diente. Mit dieser im März 1994 beendeten Recherche ist eine abgeschlossene Darstellung projektrelevanter Materialien verfügbar ${ }^{24}$. Die Universitätsbibliothek Bayreuth hat die Katalogisierung des Abschlußberichts übernommen und die Informationen nicht nur in den bayerischen Verbundkatalog, sondern auch in die einschlägigen überregionalen Datenbanken eingespeist und an die bibliothekarischen Fachorgane weitergegeben.

${ }^{24} \mathrm{Vgl}$. Die Entwicklung Bayerns durch die Integration der Heimatvertriebenen und Flüchtlinge. Gemeinsames durch das Staatsministerium für Arbeit, Familie und Sozialordnung initiiertes Forschungsprojekt der bayerischen Landesuniversitäten. Abschlußbericht der Dokumentationsstelle Bayreuth vom 15. Mai 1994, vorgelegt von Michael Reinhart (ungedrucktes Manuskript). - Anfragen sind zu richten an Prof. Dr. Rudolf Endres, Universität Bayreuth, Lehrstuhl für Bayerische Landesgeschichte, Geschwister-Scholl-Platz 3, 95440 Bayreuth. 



\section{Stefan Donth/Christian Kurzweg/ Notker Schrammek/Irina Schwab}

\section{Aufnahme und Integration von Flüchtlingen und Vertriebenen in Sachsen von 1945 bis 1952 - Vorstellung eines Forschungsprojekts}

\section{Einleitung, Forschungslage und Konzeption des Gesamtprojekts}

Der durch Massenflucht und -vertreibung aus Ostdeutschland, Ostmittel-, Ost- und Südosteuropa am Ende des Zweiten Weltkrieges ausgelöste Flüchtlingsstrom in die SBZ führte dort zu einer beispiellosen Änderung der Bevölkerungsstruktur. Die für das Land Sachsen vorliegenden Zahlen - 1949 lebten dort etwa 990000 Flüchtlinge, deren Anteil an der sächsischen Gesamtbevölkerung sich auf cirka $17 \%$ belief - deuten auf einen tiefgehenden Einschnitt in der sächsischen Bevölkerungsgeschichte hin. Allein die schon auf Grund der quantitativen Dimension naheliegende Bedeutung einer solchen demographischen Umschichtung kontrastiert auffällig mit der fast völligen Vernachlässigung dieser Thematik in der sozialgeschichtlichen SBZ/DDR-Forschung. Während in der Forschung über die westlichen Zonen die Vertriebenenproblematik schon früh einen festen Platz hatte, ist sie für die SBZ bislang noch kaum wissenschaftlich untersucht worden ${ }^{1}$. In der DDR waren Untersuchungen zur Integration der damals „Umsiedler“ genannten Vertriebenen auf dem Gebiet der SBZ/DDR erst in den letzten Jahren der SED-Herrschaft möglich. Am Magdeburger Forschungsbereich von Manfred Wille und in Berlin bei Wolfgang Meinicke entstanden Arbeiten, die sich neben der Behandlung zentraler Ereignisse auch regionalen Entwicklungen zuwandten ${ }^{2}$.

Nach dem Ende der DDR wurden die wichtigsten Archivbestände allgemein zugänglich, und politische Restriktionen für DDR-Forscher fielen weg. Inzwischen sind einige Arbeiten erschienen, die sich den Problemen der Integration von Flüchtlingen und Vertriebenen auf zonaler und regionaler Ebene widmen ${ }^{3}$ und die die Politik der „Zentralverwaltung für deutsche Umsiedler" (ZVU) untersuchen ${ }^{4}$. Daneben gerieten auch die Schicksale bestimmter Volksgruppen wie der Sudetendeutschen in den Blickpunkt des Interesses ${ }^{5}$.

Die Fülle der nun zugänglichen Quellen sowie die anfangs noch nicht voll ausgebauten Zentralstrukturen in der SBZ sprechen dafür, den Integrationsprozeß zunächst in einem

' Vgl. Krallert-Sattler, Kommentierte Bibliographie.

${ }^{2}$ Hier sei nur verwiesen auf Wille, Die Lösung der Umsiedlerfrage; Kaltenborn, Fragen der Integration sowie Meinicke, Zur Integration der Umsiedler.

3 Siehe u. a. Wille / Hoffmann / Meinicke (Hrsg.), Sie hatten alles verloren.

+ Siehe hier Schwartz, Zwischen Zusammenbruch und Stalinisierung.

${ }^{5}$ Siehe Wille (Hrsg.), Die Sudetendeutschen in der Sowjetischen Besatzungszone und Jahn (Hrsg.), Die Sudetendeutschen in Nordböhmen. 
regionalen Rahmen zu untersuchen. Gerade Sachsen kommt aus mehreren Gründen für eine solche Fallstudie in Frage: Zum einen blieb das Land in seinem territorialen Bestand weitgehend erhalten, zum anderen wiesen die sächsischen Landesbehörden in Teilbereichen eine personelle und strukturelle Kontinuität auf. Schließlich trafen die Neuankömmlinge auf ein deutlich vorhandenes sächsisches Sonderbewußtsein, dessen Bedeutung für die Aufnahmebereitschaft der alteingesessenen Bevölkerung noch nicht genügend erforscht ist. Darüber hinaus ergaben sich in Sachsen, dem am stärksten industrialisierten und urbanisierten Land der SBZ, ganz andere Schwierigkeiten bei der Integration als im vorwiegend agrarisch geprägten Mecklenburg.

Eine umfassende Erforschung dieser Problematik kann in einigen Teilbereichen an schon in der DDR entstandene Untersuchungen anknüpfen. 1985 legte Regine Just eine Arbeit vor, die sich auf der Basis von Beständen der sächsischen Archive mit der „Lösung der Umsiedlerfrage“ in Sachsen befaßte 6 . Nach dem Ende der DDR beschäftigte sich vor allem Manfred Jahn mit der Erforschung der Vertriebenenintegration in Sachsen 7 .

Diese Vorarbeiten erleichtern es, die Forschungslücke für die Integration der Vertriebenen in Sachsen zu schließen. Zu diesem Zweck fördert das Sächsische Staatsministerium des Innern an der Universität Leipzig das Projekt „Aufnahme und Integration von Flüchtlingen und Vertriebenen in Sachsen von 1945 bis 1952" unter Leitung von Prof. Dr. Ulrich von Hehl.

Die Grundüberlegung des Projektes besteht darin, nicht auf der zentralen, sondern auf der regionalen Ebene die Handlungsspielräume der politischen Kräfte zu analysieren und bei der Untersuchung der gesellschaftsgeschichtlichen Aspekte der Integration das Länderspezifische herauszuarbeiten. Dabei sollen die einzelnen Etappen des Aufnahmeund Integrationsprozesses in Sachsen für die Zeit vom Ende des Zweiten Weltkrieges bis zum Ende der Staatlichkeit Sachsens in der DDR 1952 aus der Sicht der daran beteiligten Akteure und Betroffenen untersucht werden. Neben den Beständen des Sächsischen Hauptstaatsarchivs in Dresden werden dazu Materialien der Staatsarchive in Chemnitz und Leipzig sowie ausgewählter sächsischer Kreisarchive herangezogen. Weitere wichtige Überlieferungen befinden sich im Bundesarchiv Berlin (ehemals Potsdam), in der Stiftung Archiv der Parteien und Massenorganisationen der ehemaligen DDR im Bundesarchiv Berlin, im Archiv des Deutschen Liberalismus in Gummersbach, im Archiv für Christlich-Demokratische Politik in Sankt Augustin und in verschiedenen Moskauer Archiven.

Um den unterschiedlichen Facetten der Flüchtlingsintegration in Sachsen gerecht zu werden, wurde eine Aufgliederung des Forschungsvorhabens in vier Teilprojekte vorgenommen. Neben der "Umsiedlerpolitik“ von SMAD und SED soll auch deren administrative Durchführung anhand ausgewählter Kreise in Sachsen untersucht werden. Thema einer weiteren Arbeit ist der Beitrag der sächsischen LDP zur Integration der Flüchtlinge und Vertriebenen. Eine alltags- und mentalitätsgeschichtliche Studie zum Selbstbild und zu den Erfahrungsstrukturen der Vertriebenen ergänzt die politik- und sozialgeschichtlich geprägten Teilprojekte, die im folgenden vorgestellt werden.

6 Siehe Just, Lösung der Umsiedlerfrage.

7 Siehe Jahn, Auffang- und Quarantänelager, sowie ders., Veränderungen wirtschaftlicher und sozialer Strukturen in Sachsen. 


\section{Einfübrung in die Teilprojekte}

2.1. Der Einfluß von SMAD und SED auf Aufnahme und Integration der Flüchtlinge und Vertriebenen in Sachsen 1945 bis 1952 (Stefan Donth)

In diesem Teilprojekt werden die Grundsatzentscheidungen der Flüchtlings- und Vertriebenenpolitik der sowjetischen Besatzungsmacht und der SED, der beiden wichtigsten Entscheidungsträger in der SBZ/DDR, untersucht. Um die in einem komplexen Geflecht von Macht- und Einflußstrukturen eingebetteten Entscheidungsspielräume der Akteure in Sachsen aufzeigen zu können, ist es notwendig, sowohl die Entwicklungen auf der zentralen Ebene und deren Auswirkungen auf die Tätigkeit der sächsischen Behörden als auch deren eigene Politik zu betrachten. Vieles deutet darauf hin, daß in der SBZ letztendlich die Vorgaben der sowjetischen Besatzungsmacht maßgebend waren. Deshalb soll analysiert werden, in welchem Ausmaß und mit welchen Methoden die Behörden der SMAD in Berlin, die ZVU und die SED-Führung die Integration der Vertriebenen in Sachsen beeinflußten. Da auf diesem Gebiet in der Anfangszeit der SBZ oft wichtige Entscheidungen noch in den Ländern fielen und auch von diesen umgesetzt werden mußten, weil zentrale Apparate zu diesem Zeitpunkt dazu nur begrenzt in der Lage waren, soll untersucht werden, welchen Spielraum die in Dresden angesiedelten Instanzen der SMA, SED und die Landesregierung in den ersten Nachkriegsjahren besaßen und in welchem Ausmaß sich dieser in den späten vierziger Jahren durch die fortschreitende Zentralisierung und den verstärkten Ausbau der Zentralverwaltungen und danach der DWK immer mehr verringerte. Die Untersuchung der Politik auf der Landesebene ist auch deshalb von besonderer Bedeutung, weil wesentliche Maßnahmen wie die Bereitstellung von Wohnraum und Arbeitsplätzen durch die Umsiedlerabteilung der sächsischen Landesregierung erfolgten. Deren Tätigkeit bestimmten jedoch in entscheidendem Maße die Befehle und Vorgaben der verschiedenen Dienststellen der SMAS, die sich nicht auf die Regelung von Grundsatzentscheidungen beschränkte, sondern auch deren Umsetzung durch die Landesregierung kontrollierte. Das umfaßte, angefangen von der Organisation der Transporte aus den Vertreibungsgebieten, der Einrichtung der sogenannten Umsiedlerlager bis hin zu politischen, sozialen und wirtschaftlichen Fragen, alle Bereiche der Integration der Vertriebenen in Sachsen ${ }^{8}$.

Eine umfassende Analyse der politikgeschichtlichen Aspekte dieses Prozesses in Sachsen, die nur unter Einbeziehung der zonalen und der regionalen Akteure erfolgen kann, wird aber unvollständig bleiben, wenn nicht die Politik der SED gegenüber den Vertriebenen betrachtet wird. Dabei erhärten erste Archivrecherchen die Annahme, daß den „Umsiedlern" im Vergleich zu anderen Fragen und Problemen im Rahmen der Gesamtpolitik der SED nur eine untergeordnete Bedeutung zukam. Das betraf nicht nur die Stellung der mit der Vertriebenenintegration befaßten Abteilungen und Mitarbeiter im zentralen Parteiapparat in Berlin und in der sächsischen SED-Landesleitung; auch auf wichtigen Konferenzen und Beratungen der SED auf Zonen- und Landesebene wurde dieses Thema oft nur am Rande angesprochen. Ungeachtet dessen, daß „Umsiedlerfragen“ in der Gesamtpolitik der SED keinen herausgehobenen Platz einnahmen, prägte die Partei doch neben der Besatzungsmacht in hohem Maße die Integrationspolitik in der SBZ/DDR.

${ }^{8} \mathrm{Zu}$ diesem Fragekomplex wurden jetzt sowjetische Archivalien untersucht. Siehe hier vor allem Naimark, The Russians in Germany. 
Dabei müssen für Sachsen mehrere Ebenen der Einflußnahme der SED unterschieden werden, denn die Partei lenkte in engem Zusammenwirken mit der SMA die Tätigkeit der Landesregierung, unterstützte deren Arbeit durch eine intensive Propagandatätigkeit und versuchte parallel dazu, die Vertriebenen in das politische System der SBZ/DDR einzubinden.

Es war von nicht zu unterschätzender Bedeutung, daß die SED die Umsetzung der Befehle und Maßnahmen der Besatzungsmacht vor allem durch ihre Personalpolitik in den für die Vertriebenen zuständigen Ministerien und Abteilungen der Landesregierung garantierte. Anfang 1947 waren von den 34 Angestellten der „Abteilung für deutsche Umsiedler" in der Landesregierung Sachsen 32 Mitglieder der SED, einer Mitglied der LDP und einer parteilos. Als die Liberaldemokratin Ruth Fabisch Mitte 1947 die Leitung der Abteilung übernahm, änderte das nichts am bestimmenden Einfluß der SED. Damit war die Partei in der Lage, die Arbeit der Verwaltung zu steuern, die die für große Teile der alteingesessenen Bevölkerung oft harten Entscheidungen in den Verteilungskämpfen der Nachkriegszeit durchsetzte und damit einen Teil des Unmutes und der Kritik von der SED fernhielt ${ }^{9}$.

Die SED beschränkte sich jedoch nicht auf eine Überwachung und Steuerung der Arbeit der Landesregierung, sondern unterstützte diese in vielen Fällen durch eine intensive Propaganda und mehrere Kampagnen. Daran kann die Stellung der SED im Machtgefüge der SBZ vor allem im Vergleich zur Verwaltung untersucht werden. Ausgehend von den Planungen der SED und dem Stellenwert der Vertriebenen in diesen Maßnahmen ist zu fragen, welchen Anteil die SED an der Verbesserung des Lebensstandards dieser Bevölkerungsgruppe besaß und in welchen Bereichen sich deshalb eine Zunahme des politischen Einflusses der SED beobachten läßt. Beispielsweise versuchten die sächsische Landesregierung und die Führungsgremien von KPD bzw. SED bei der Vorbereitung und Durchführung der Bodenreform, die Vertriebenen bei der Landzuteilung und dem späteren Ausbau ihrer Neubauernwirtschaften zu unterstützen, um die relativ schwache Position von KPD/SED unter der Landbevölkerung durch die Hinzugewinnung dieser Gruppe als neuer Klientel zu verbessern. Da die zur Verfügung stehenden materiellen Ressourcen begrenzt waren und weil die für deren Verteilung zuständige VdgB sich nicht immer instrumentalisieren ließ, konnte die KPD/SED hier nur in regional unterschiedlichem Ausmaß ihre politische Basis gezielt erweitern ${ }^{10}$.

Ein weiteres Aufgabengebiet der SED bestand darin, die örtlichen und überregionalen Behörden sowie die eigene Mitgliedschaft für die Probleme der „Umsiedler“ zu interessieren. Dabei stand die SED oft vor schwierigen Entscheidungen, denn sie mußte garantieren, daß die Konflikte bei der Eingliederung der Vertriebenen sowohl von Seiten der „Umsiedler“ als auch der Alteingesessenen, die die Mehrheit der Bevölkerung stellten, nicht eskalierten. Die Untersuchung der Politik der SED in den mit der Integration der Vertriebenen befaßten Gremien soll aufzeigen, in welchem Ausmaß sich die Partei hier zugunsten dieser Gruppe positionierte und den Integrationsprozeß politisch absicherte.

Dafür war die Umsetzung der Vorgaben der Parteiführung durch die Parteiorganisationen vor Ort und die maßgebenden Funktionäre entscheidend, denn von deren Verhalten in den Konflikten zwischen alteingesessener Bevölkerung und den „Umsiedlern“

"Siehe BAB, DO 1/10/2, Bl. 159-160.

$10 \mathrm{Vgl}$. dazu als ersten Hinweis den „Bericht über den Stand der Organisation im Bezirk Dresden vom 15. 11. 1945“ der KPD, in: SAPMO, RY1/13/8-10/186, Bl. 43. 
hing es oft $a b$, welche Position beide Bevölkerungsgruppen zur SED einnahmen. Es läßt sich auf vielen Gebieten nachweisen, daß es der Partei vor allem darum ging, ihre Basis unter den Vertriebenen zu verbreitern, konkurrierende Vorstellungen insbesondere der bürgerlichen Parteien und der Kirchen einzubinden und damit deren Einfluß auf diese Bevölkerungsgruppe zu begrenzen. Das wird besonders gut sichtbar, wenn man die Politik der SED im Jahr 1947 untersucht, als sie mit den „Umsiedlerausschüssen“ in der gesamten SBZ versuchte, durch eine Mobilisierung der politischen Kräfte der SBZ eine Verbesserung der Lage der "Umsiedler" zu erreichen. Zumindest in Sachsen reagierte die Partei damit nicht nur auf das Unvermögen der Landesregierung, das „Umsiedlerproblem" zu lösen, sondern wollte damit auch ihre Kompetenz unterstreichen und sich zum wichtigsten Fürsprecher für die Interessen der Vertriebenen aufwerten. Das erschien der sächsischen SED-Führung auch deshalb als notwendig, weil sowohl CDU als auch LDP mit jeweils eigenen Initiativen im Landtag deren Notlage angesprochen hatten ${ }^{11}$.

Von ähnlichen Motiven ließ sich die KPD/SED auch bei der Arbeit der Volkssolidarität, einem ihrer wichtigsten Instrumente bei der Lösung der sozialpolitischen Aufgaben der Nachkriegszeit, leiten. Hier wurde versucht, durch das Prinzip der Freiwilligkeit der Spenden die für die Verteilung an Vertriebene und andere Bedürftige notwendigen Mittel zu beschaffen, ohne dabei durch Zwangsmaßnahmen zusätzliche Konflikte mit der alteingesessenen Bevölkerung zu provozieren. Die Verteilung von Spenden ließ sich zudem gut für eigene Propagandazwecke ausnützen ${ }^{12}$. Ob es der Partei damit gelang, große Teile der Vertriebenen an sich zu binden und für den Aufbau einer neuen Gesellschaftsordnung zu mobilisieren, kann beispielsweise anhand der Vorbereitung und Durchführung der Umsiedlerwoche „Neue Heimat - Neues Leben“ untersucht werden, als die SED gezielt auf ihren Beitrag zur Verbesserung der Lage der Vertriebenen hinzuweisen versuchte.

Nach Meinung der SED war es für die Integration der Vertriebenen entscheidend, daß sich diese mit der Endgültigkeit des Verlassens ihrer Heimat und mit den Nachkriegsgrenzen Osteuropas abfanden. Das glaubte die Partei am besten durch die entschlossene Verhinderung jeglicher landsmannschaftlicher Gruppenbildung und die ständige Verbesserung des Lebensstandards der "Umsiedler" erreichen zu können. Damit sollte auch der unter den Vertriebenen vorhandene Unmut über die Oder-Neiße-Grenze abgebaut werden. Für eine Beurteilung des Ergebnisses der Integration ist es besonders wichtig, die Auswirkungen der Ansiedlung der Vertriebenen auf die Wirtschaftsstruktur und den Arbeitsmarkt Sachsens zu untersuchen. Das muß auch eine Analyse darüber einschließen, in welchen Wirtschaftszweigen und Berufen Vertriebene überproportional vertreten waren ${ }^{13}$. Eine Verbindung von politik- und sozialgeschichtlichen Fragestellungen ist auBerdem notwendig, wenn der enge Zusammenhang zwischen der Kampagne um die Anerkennung der „Oder-Neiße-Friedensgrenze“ und dem „Gesetz über die weitere Verbesserung zur Lage der ehemaligen Umsiedler in der DDR" vom 8. September 1950 dargestellt werden soll.

${ }^{11}$ Siehe dazu beispielsweise das Protokoll der 6. Sitzung des Sächsischen Landtages vom 17. 1. 1947, in: Akten und Verhandlungen des Sächsischen Landtages 1946-1952, Band I.1. Vgl. auch den Beitrag von Christian Kurzweg.

12 Diese Überlegungen fanden schon früh Eingang in die politische Diskussion in Sachsen. Siehe dazu das Protokoll der Sitzung des erweiterten Landesvorstandes der SPD vom 18. 12. 1945, in: SächsHStA, SED-BPA Dresden, II/A/1.001.

13 Siehe hier auch den Beitrag von Irina Schwab. 
Der Kampagne zur Anerkennung der osteuropäischen Nachkriegsgrenzen wurde durch die SED-Führung ein hoher Stellenwert beigemessen, denn zum einen waren die Gebietsverluste im Osten bis in die Reihen der eigenen Mitgliedschaft hinein unpopulär, zum anderen bestanden unmißverständliche Vorgaben der Besatzungsmacht, daß die Grenzen nicht zur Disposition stünden. Die SED, die befürchtete, daß große Teile der Vertriebenen unter „reaktionären“ Einfluß geraten könnten, mußte deshalb auf Anweisung der SMAD den „Umsiedlern“ suggerieren, daß nur sie als eine starke Partei für eine erfolgreiche Revision der Grenzfrage eintreten könnte und auf diese Weise versuchen, große Teile dieser Bevölkerungsgruppe an die Partei zu binden. Nach der Gründung der DDR 1949 und ihrer Anerkennung der Oder-Neiße-Grenze 1950 beschied die SED den „Umsiedlern“, bei raschen Fortschritten beim Aufbau des Sozialismus in der DDR würde die Grenze viel von ihrer Härte verlieren. Eine Analyse der Politik der sächsischen SED-Führung wird der Frage nachgehen müssen, mit welchen Methoden eine Akzeptanz der neuen Grenze innerhalb der eigenen Mitgliedschaft und den Vertriebenen erreicht werden sollte. Hier ist eine Verbindung mit dem schon erwähnten „Umsiedlergesetz" von 1950 zu vermuten, denn der Propaganda für die Oder-Neiße-Grenze sollte durch eine gezielte Verbesserung der Lebensbedingungen der Vertriebenen eine höhere Durchschlagskraft verliehen werden. Eine Untersuchung der Genese dieses Gesetzes wird die tatsächlichen Lebensumstände der „Neubürger“ und die spezifischen sächsischen Interessen im Rahmen des Gesetzgebungsprozesses in den Blick nehmen müssen. Dabei ist vor allem zu klären, in welchem Umfang die Vertriebenen bei der Zuteilung von Wohnraum und Haushaltsgegenständen gegenüber der alteingesessenen Bevölkerung benachteiligt waren, denn die "Wohnungsfrage" galt in den Augen der SED-Führung als ein wichtiges Kriterium für den Erfolg der Seßhaftmachung der „Umsiedler“. Die SED warb mit dem „Umsiedlergesetz“ auch um die Zustimmung der Vertriebenen zur Einheitsliste bei den ersten Volkskammerwahlen 1950. Als nach den Wahlen die Umsetzung der gesetzlichen Vorgaben durch die DDR-Regierung eingeschränkt wurde und nur ein geringer Teil der „Umsiedler“ in den Genuß der versprochenen Maßnahmen kam, rief das Verbitterung und Enttäuschung unter den Vertriebenen hervor und vergrößerte die Ablehnung des Regimes unter ihnen. Daß sich Besatzungsmacht, SED und Verwaltung insbesondere vor Wahlen und Abstimmungen für die Situation der Vertriebenen interessierten und bestrebt waren, deren Zustimmung zu erreichen, läßt sich für den gesamten Zeitraum von 1945 bis 1952 nachweisen. Eine Untersuchung des Wahlkampfes der SED in Hinblick auf die „Umsiedler" muß mit einer Analyse des Wahlverhaltens der Vertriebenen verbunden werden, weil dadurch Rückschlüsse darauf möglich sind, in welchem Ausmaß die SED von den Vertriebenen als eine Vertreterin ihrer Interessen begriffen wurde.

\subsection{Die administrative Umsetzung der Integration von Flüchtlingen und Vertriebenen} in Sachsen von 1945 bis 1952 (Irina Schwab)

Der zweite Teil des Projekts beschäftigt sich mit der administrativen Umsetzung der Flüchtlingsintegration durch die mittlere und untere Verwaltungsebene, denn die Behörden der Kreise und Orte trugen die Hauptlast der Unterbringung der Vertriebenen und ihre Ausstattung mit den nötigsten Hausratsgegenständen. Deshalb wird die Umsetzung der Vorgaben der Landesregierung am Beispiel ausgewählter Kreise untersucht. Im Mittelpunkt stehen Maßnahmen der Kreisverwaltungen bei der Aufnahme der Vertriebenen 
in den Ansiedlungsorten, die mittelfristigen Hilfen für die „Neubürger“ sowie deren Integration durch die Bereitstellung von Wohnraum und Arbeitsplätzen. In zahlreichen Anleitungen, Rundverfügungen und sonstigen Schreiben der Landesregierung wurden Landräte und Bürgermeister immer wieder auf die Wichtigkeit dieser Aufgaben hingewiesen, ihre persönliche Verantwortung wurde dabei hervorgehoben ${ }^{14}$. In diesem Zusammenhang stellt sich die Frage, ob sich die Parteizugehörigkeit der Verantwortlichen auf Kreis-, aber auch auf Gemeindeebene auf ihre Bemühungen bei der Lösung der Probleme der Vertriebenenintegration niederschlug, doch ist auch die Mitarbeit der Vertriebenen in den entsprechenden Ausschüssen und Behörden ins Auge zu fassen.

Da die Erforschung des Themas auf Grund des umfangreichen Materials nicht alle sächsischen Kreise umfassen kann, mußte eine Auswahl getroffen werden. So wird, stellvertretend für den überwiegend landwirtschaftlich geprägten Norden des Landes, die Entwicklung in den Kreisen Leipzig-Land und Grimma mit der des Kreises Glauchau verglichen, der mit seiner Textilindustrie im durch die Industrie dominierten Südwesten Sachsens liegt. Hierbei muß auch das Problem der unterschiedlichen Integrationsmöglichkeiten in dörfliche oder städtische Strukturen beachtet werden. Um zu repräsentativen Ergebnissen zu gelangen, wird ferner der Grenzkreis Bautzen in die Betrachtung mit einbezogen, da sich die Kreise entlang der südlichen und östlichen Grenze des Landes Sachsen besonderen Schwierigkeiten ausgesetzt sahen, denn sie waren Einfallstor der Flüchtlingsströme, deren erste Aufnahme besonders in der Anfangszeit eine nahezu unlösbare Aufgabe zu sein schien. In der Folgezeit waren diese Regionen begehrter Ansiedlungsort der Vertriebenen. Schließlich werden Untersuchungen zu den Kreisen Aue und Leipzig-Stadt durchgeführt, denn Aue war auf Grund des forcierten Erzbergbaus der Wismut spezifischen Wohnraum- und Arbeitsmarktproblemen ausgesetzt und die Messe- und Universitätsstadt Leipzig, zugleich größte Stadt Sachsens, nahm rein zahlenmäßig die meisten Vertriebenen auf.

Zunächst werden das Verhalten und die Maßnahmen der mittleren Verwaltungsebene bei der Aufnahme der ihr von der Landesregierung zugewiesenen Kontingente der Vertriebenen untersucht. Dazu müssen vor allem zwei Fragen im Mittelpunkt stehen: die Spielräume der Kreisbehörden gegenüber dem Land sowie die Verteilung der Verantwortlichkeiten auf beiden Seiten. Hierbei ist zu klären, in welchem Ausmaß die Bestrebungen der Landesverwaltung bzw. Landesregierung Sachsen, die Unterbringung und Integration der Vertriebenen zentral zu koordinieren, sich in der Tätigkeit der Kreisverwaltungen widerspiegelten und ob sich das Problem auch auf die untere Ebene, die Gemeinden, auswirkte.

Eine Untersuchung der Tätigkeit der mittleren Verwaltungsebene ist auch deshalb wichtig, weil diese bereits vor der Arbeitsaufnahme der Landesverwaltung Sachsen am 9. Juli 1945 vor den Problemen des nicht abreißenden Flüchtlingsstromes standen und die Kreisverwaltungen mit bisher nicht gekannten Anforderungen bei der Unterbringung und Versorgung von ungefähr einer Million Menschen bei einer am Boden liegenden Wirtschaft und einer sich ständig verschärfenden Wohnungsnot fertig zu werden hatten. $\mathrm{Da}$ in der Anfangszeit die Koordinationsversuche übergeordneter Organe nur sehr langsam spürbar wurden, versuchte jeder Landrat oder Bürgermeister, allein mit diesen Problemen fertig zu werden; das geschah oft auf Kosten der Nachbarkreise oder -orte. Erst

${ }^{14}$ Vgl. z. B. SächsHStA, LRS, MdI 2190, Verfügung der ZVU v. 30. 10. 45 an die Ämter für Umsiedler bei den Länder- und Provinzialverwaltungen, Landräte und Bürgermeister der SBZ. 
mit der Etablierung des Amtes für deutsche Umsiedler bei der Landesregierung Sachsen im Zusammenhang mit der Gründung der ZVU am 25. September 1945 wurde eine bessere Koordination der Arbeit innerhalb des Landes, aber auch zwischen den einzelnen Ländern der SBZ möglich. Dennoch gab es auch lange danach Rivalitäten zwischen den Ländern wie zwischen den Kreisen und Orten in Bezug auf Weiterleitung und Aufnahme der Vertriebenen. Jede Seite glaubte sich angesichts der gewaltigen Flüchtlingsmassen und der damit verbundenen Schwierigkeiten bei den Zuteilungen der aufzunehmenden Menschen gegenüber den anderen Orten, Kreisen oder Ländern benachteiligt.

Eine Untersuchung der Tätigkeit der Kreisverwaltungen wird sich in diesem Zusammenhang vor allem damit beschäftigen müssen, in welchem Maße Landräte und Bürgermeister Zentralisierungsbestrebungen der Landesregierung und der ZVU begrüßten, um die Probleme bei der Aufnahme und Integration der Vertriebenen nicht mehr allein lösen zu müssen und ob und in welchem Ausmaß die mittlere Verwaltungsebene bestrebt war, sich eigene Spielräume in der Umsiedlerpolitik zu erhalten. Daneben muß in die Untersuchung einbezogen werden, wie die Aufnahme auf der Ebene der Kreise verlief, denn sowohl Besatzungsmacht als auch SED und Landesregierung forderten eine rasche Assimilierung der Vertriebenen mit der ansässigen Bevölkerung. Deshalb sollte der Eindruck der Neuankömmlinge von ihrer "neuen Heimat" von Anfang an so günstig wie möglich sein. Dazu gehörte es auch, eventuelle Widerstände in der alteingesessenen Bevölkerung gegen die Aufnahme der Vertriebenen zu unterbinden ${ }^{15}$. Außerdem hatten die Behörden der Kreise nach Abschluß des Aufnahmeprozesses besondere Bedeutung bei der Leistung von mittelfristigen Hilfen für die Vertriebenen. Das betraf die Auszahlung der Umsiedlerbeihilfen, die Ausstattung der zugewiesenen Wohnräume mit den notwendigsten Hausratsgegenständen und die Zuweisung erster Arbeitsplätze. Für diese Fragestellungen muß die Tätigkeit der Umsiedlerausschüsse in die Untersuchung der Politik der Behörden der Kreise mit einbezogen werden. Diese standen den Umsiedlerämtern als beratende Organe zur Seite und versuchten, die Behörden für die Probleme der „Umsiedler" zu sensibilisieren. Die Umsiedlerausschüsse sollten sich neben Vertretern der Parteien und Massenorganisationen aus Vertriebenen und Einheimischen zusammensetzen, um auch dadurch den Integrationsprozeß zu fördern. Ebenfalls muß im Zusammenhang mit der Unterbringungsfrage auf die Wohnungsämter und die Wohnungsausschüsse eingegangen werden. Letztere existierten häufig sogar als gemischte Ausschüsse zu Umsiedlerund Wohnungsfragen, was die enge Verknüpfung beider Bereiche zeigt ${ }^{16}$.

Ein weiterer Komplex beschäftigt sich mit der Frage, wie die Zusammenarbeit zwischen der Landesverwaltung/Landesregierung Sachsen und der SMAD bzw. der SMAS auf mittlerer Verwaltungsebene verlief, wie die Landesregierung Anordnungen an die einzelnen Kreise weiterleitete und wie diese dann schließlich in den Städten und Gemeinden umgesetzt wurden. Natürlich sind hierbei auch die Kontrollmechanismen sowohl der sowjetischen Militärbehörden als auch der Landesregierung und der Kreise gegen-

${ }^{15}$ So brachte etwa eine Kontrollfahrt von Vertretern der LRS in den Kreis Meißen am 10. 7. 1947 das Ergebnis, daß es laut Aussagen der dortigen Behörden Widerstände in der Bevölkerung gegen die Zuweisung von Vertriebenen gab. Vgl. SächsHStA, LRS, MdI 2659, Schreiben LRS Ministerium für Arbeit und Sozialfürsorge Hauptabteilung für deutsche Umsiedler an Kreisrat zu Meißen 30. 7. 1947.

16 So waren im Kreis Glauchau Umsiedler- und Wohnungsausschuß miteinander verknüpft. Vgl. SächsHStA, KT/KR Glauchau 46, unpag., Niederschrift der 4. Sitzung des Hauptausschusses des Kreistages vom 19. 3. 1948. 
über den jeweils untergeordneten Stellen von Bedeutung. Vor allem war man der Besatzungsmacht rechenschaftspflichtig, die im Falle einer ungenügenden Lösung der anstehenden Probleme Druck auf die entsprechenden deutschen Behörden ausübte.

Um diese Fragen zu klären, liegt es nahe, mit der Erforschung der Zuteilung der ankommenden Flüchtlinge und Vertriebenen zu ihren neuen Wohnorten zu beginnen. Das Land Sachsen war zu Beginn der ersten "regulären" Vertreibung nach dem Potsdamer Abkommen, abgesehen von der Zuteilung einiger Facharbeiter für die Industrie, lediglich als Transitland nach den anderen Ländern der SBZ bzw. den westlichen Besatzungszonen bestimmt. Der von der SMAD festgelegte Verteilerschlüssel sah hauptsächlich die Länder Mecklenburg und Brandenburg als Ansiedlungsgebiete für die Vertriebenen vor. Aber angesichts der gewaltigen Flüchtlingsströme, die sich schon vor Kriegsende und während des gesamten Jahres 1946 in die SBZ ergossen, war das nicht durchzuhalten, obwohl Sachsen vor allem im Spätsommer 1945, bevor andere Anweisungen eingingen, auch mit radikalen Mitteln versuchte, das Land von den Vertriebenen zu räumen und sie in die für sie bestimmten Länder zu transportieren. Dort wurde ihnen oftmals die Aufnahme verweigert, und ihre erzwungene Rückkehr verstärkte das überall herrschende Chaos ${ }^{17}$.

Eine der wichtigsten Aufgaben der Behörden der Kreise bestand in der Aufteilung der ihnen von SMA und Landesregierung zugewiesenen Vertriebenen auf die einzelnen Gemeinden, die dann für die Unterbringung in Privatquartieren zu sorgen hatten. Die damit verbundenen administrativen Vorgänge lassen sich anhand des Aktenmaterials sehr gut rekonstruieren und zeigen einen weit verbreiteten Widerstand untergeordneter Organe gegen die Zuteilungen von Vertriebenen. Das äußerte sich beispielsweise in den überlieferten Schriftwechseln zwischen den zuständigen Kreisbehörden und der Landesregierung, in denen wechselseitig der Vorwurf der Ungerechtigkeit bzw. des unkorrekten Arbeitens erhoben wurde. Auch war es nicht unüblich, die in den Orten ankommenden Menschen längere Zeit in Sammellagern unterzubringen, bevor eine Einweisung in Wohnungen möglich war. Hiergegen gingen SMA und Landesregierung entschieden vor. Sie wiesen die Kreisverwaltungen immer wieder darauf hin, daß die Wohnraumstatistiken die Grundlage der errechneten Verteilerschlüssel seien, die in Form von Wohnraumkarteien bei den Wohnungsämtern geführt wurden. Daß auf eine gerechte Aufteilung des vorhandenen Wohnraumes unter der gesamten Bevölkerung von Seiten der SMA und der Landesregierung besonderer Wert gelegt wurde, läßt sich auch daran zeigen, daß die Wohnraumfrage noch vor der Eingliederung der Ankömmlinge in den Arbeitsprozeß stand. Die Wohnungsämter der Kreise und Orte sowie deren Wohnraumstatistiken waren das Ziel der von der SMA häufig geforderten Kontrollfahrten durch Kommissionen der Landesregierung, aber auch der Kreise in die Gemeinden. Dadurch sollte vermieden werden, daß die Kreise durch falsche, nicht dem Kontrollratsgesetz Nr. 18 entsprechende Wohnraumberechnungen eine Erleichterung in der Zuteilung der Vertriebenen erzielten oder zu Sperrgebieten für den Zuzug erklärt wurden. Solche Versuche lassen sich häufig nachweisen ${ }^{18}$.

17 Vgl. u. a. SächsStA Leipzig, KT/KR Grimma 182, Bl. 61f, Rundverfügung LVS Ressort Inneres und Volksbildung 10. 8. 1945 (zur Lösung des Flüchtlingsproblems in Sachsen) Dieses Schreiben besagt, daß bis zum 31.8. 1945 cirka 2 Millionen Flüchtlinge aus Sachsen herausgeschafft werden müßten.

18 Vgl. SächsHStA, KT/KR Bautzen 134, unpag., Niederschrift 2. Sitzung Kreisneubürgerausschuß 10. 4. 1947, KT/KR Bautzen 887, unpag., Bericht Kontrollfahrt Landkreis Bautzen 18.-26. 3. 1947, KT/KR Bautzen 57, unpag., Niederschriften Sitzungen Vorstand des Kreistages 1947. 
Besonders aufschlußreich sind die Bemühungen der Kreisverwaltungen, die meist völlig mittellos ankommenden Vertriebenen mit Hausrat, Heizmaterial, Kleidung und Schuhwerk zu versorgen. Die durch die SMA genehmigten Waren- und Materialzuteilungen waren in den meisten Fällen nur ein Tropfen auf den heißen Stein, so daß die Kreisverwaltungen eigene Anstrengungen unternehmen mußten, um den größten Notständen abhelfen zu können. Da die durch Spendenaktionen der Volkssolidarität aufgebrachten Mittel völlig ungenügend waren, griffen zahlreiche Gemeinden mit eigenen Ortsgesetzen zu Zwangsmaßnahmen. Deren Ergebnisse sind noch genauer daraufhin zu analysieren, in welchem Maße Ressourcen der alteingesessenen Bevölkerung für die Vertriebenen nutzbar gemacht werden konnten.

Selbst das schon erwähnte "Umsiedlergesetz" vom 8. September 1950, das Hilfe für Umsiedler-Neubauern, Ausbildungsbeihilfen für die Kinder der Vertriebenen, sowie Umsiedlerhandwerker- und Wohnbedarfskredite versprach, konnte die schwierige materielle Lage dieser Bevölkerungsgruppe nicht lindern. Die Einschränkung des Wohnbedarfskredites nach den Volkskammerwahlen vom 15. Oktober 1950 löste großen Unmut unter den Betroffenen aus, was sich in einer Reihe von Beschwerden niederschlug. Nachdem sich zu Beginn der fünfziger Jahre die Wohnraumsituation etwas zu entspannen begann und die Vertriebenen, die zumeist in Untermietsverhältnissen lebten, aus den immerhin spärlich möblierten Zimmern herausgenommen wurden, standen sie in den neu zugeteilten größeren Wohnungen wiederum ohne Mobiliar da. Das war wohl auch einer der Gründe, daß es immer wieder Fälle gab, in denen sich Vertriebene weigerten, aus den Notwohnungen und Barackenunterkünften auszuziehen ${ }^{19}$. Überhaupt war die Situation vieler Vertriebener noch zu Beginn der fünfziger Jahre schwierig. Man kann davon ausgehen, daß es im allgemeinen keine Gleichstellung im Lebensstandard mit der altansässigen Bevölkerung gegeben hat. So kam es beispielsweise häufig vor, daß die Wohnbedarfskredite zweckentfremdet für die Anschaffung von Bekleidung verwendet wurden. Die Integrationsmaßnahmen und mittelfristigen Hilfen bis zum Ende der vierziger Jahre, aber auch das "Umsiedlergesetz" brachten nur in begrenztem Maße die erwarteten Ergebnisse.

2.3. Bürgerliche Parteien und Vertriebene: Zur „Umsiedlerpolitik“ der sächsischen LDP (Christian Kurzweg)

Untersuchungsgegenstand des dritten Teilprojektes ist die Politik der bürgerlichen Parteien. Dabei soll der Schwerpunkt auf der LDP liegen, die sich - anders als die CDU ausdrücklich als "nichtsozialistisch“ verstand. Besonderes Interesse verdient der Umgang der LDP mit dem Vertriebenenproblem auch deshalb, weil die Partei bis 1949 auf die Festschreibung programmatischer Grundsätze mit der Begründung verzichtete, sich ganz auf die Tagesaufgaben konzentrieren zu wollen ${ }^{20}$.

Die Frage nach dem konkreten Beitrag der bürgerlichen Parteien der SBZ zur Integration der Flüchtlinge und Vertriebenen scheint auf den ersten Blick wenig ertragreich zu sein. Auf die alliierte Zuständigkeit für die Regelung völkerrechtlicher Fragen gestützt, behielt sich die sowjetische Besatzungsmacht besonders auf diesem Gebiet die Grund-

${ }^{19}$ Vgl. u. a. SächsStA Leipzig, KT/KR Leipzig 107, Bl. 57, Niederschrift 7. Sitzung Rat des Kreises 15. 2. 1952.

${ }^{20}$ Vgl. u. a. Dähn, LDP; Dirksen, Bürgertum. 
satzentscheidungen vor, zumal die Vertreibungsproblematik heikle Fragen wie das Verhalten der Roten Armee in den letzten Kriegsmonaten oder die territoriale Neugestaltung Polens berührte ${ }^{21}$. Da die Zwangsumsiedlungen als unumkehrbar und endgültig angesehen wurden, rückte eine rasche und nachhaltige Assimilation der Betroffenen, verstanden vor allem als sozialökonomische Gleichstellung mit der alteingesessenen Bevölkerung, in das Zentrum der Vertriebenenpolitik. Deren Koordinierung und detaillierte Ausgestaltung wurde von der Sowjetischen Militäradministration zum einen auf die $\mathrm{KPD}$, dann die SED, zum anderen auf die Zentral- und Landesverwaltungen übertragen. Ein Blick auf die Strukturen, die Funktionsabläufe und die Zusammensetzung der Verwaltungen in der SBZ zeigt, daß der Einfluß der bürgerlichen Parteien auf die Versorgung mit Wohnraum, die soziale Absicherung und die Vermittlung von Arbeitsplätzen nur mittelbar sein konnte. Die SED besaß ihnen gegenüber einen deutlichen Informations- und Ressourcenvorsprung und die Möglichkeit der Rückversicherung bei der Besatzungsmacht, zudem waren die maßgeblichen Positionen in der Regel mit kommunistischen Funktionären besetzt. Dem Personal der ZVU gehörte überhaupt kein Mitglied einer bürgerlichen Blockpartei an ${ }^{22}$.

Eine solche Sichtweise nähme allerdings die „einseitig ausgerichtete und politisch determinierte Integrationsdoktrin ${ }^{\text {“23 }}$ der Besatzungsmacht und der SED als alleinigen Maßstab und würde zum Beispiel bestimmte soziokulturelle Aspekte ausklammern: Während die Forschung inzwischen das ausgeprägte Desinteresse der SED an der kulturellen und historischen Identität der Vertriebenen herausgearbeitet hat, ist über die Haltung der bürgerlichen Parteien in dieser Hinsicht kaum etwas bekannt. Ähnlich verhält es sich mit der Frage, welchen Einfluß diese Parteien auf die Aufnahmebereitschaft der Alteingesessenen nahmen, besonders dort, wo ihre Klientel überproportional stark mit den materiellen und immateriellen Bedürfnissen der Neuankömmlinge konfrontiert wurde. Hier waren Konflikte vorgezeichnet, da LDP und CDU auch von Vertriebenen häufig als Interessenvertreter in Anspruch genommen wurden. Zu diesem Umstand trug bei, daß die SED die sogenannten "Neubürger" u. a. ihrer sozialen Herkunft, starker religiöser Bindungen und „politischer Passivität" wegen als Unruhefaktor im gesellschaftlichen Umgestaltungsprozeß betrachtete und ihnen mit Mißtrauen gegenüberstand ${ }^{24}$. Die berufliche und soziale Eingliederung ging deshalb mit einer permanenten ideologischen Beeinflussung einher, die antisowjetische und antikommunistische Einstellungen beseitigen und die Vertriebenen systemkonform politisieren sollte ${ }^{25}$. Ob die LDP von den Betroffenen als Opposition zur SED wahrgenommen wurde, steht allerdings noch in Frage. In diesem Zusammenhang müssen die in der Partei vorhandenen Vorbehalte gegenüber den Vertriebenen hinsichtlich ihre Motivation, Verbreitung und etwaigen Veränderung genauer erkundet werden.

Als offenkundig politische Hegemonie beanspruchende und eng mit der Besatzungsmacht liierte Partei wurde hauptsächlich die SED für Versäumnisse und Fehlentscheidungen bei den Integrationsbemühungen verantwortlich gemacht. Daher gab es Überlegungen, sich vom „Eindruck der Alleinzuständigkeit“ zu entlasten und die Blockparteien

${ }^{21}$ Vgl. u. a. Henke, Weg nach Potsdam, S. $63 \mathrm{ff}$.

${ }^{22}$ Siehe Schwartz, Zwischen Zusammenbruch und Stalinisierung, S. 50.

${ }^{23}$ Hoffmann / Wille / Meinicke, Flüchtlinge und Vertriebene, S. 17.

${ }^{24}$ Vgl. Staritz, Gründung der DDR, S. $64 \mathrm{f}$.

${ }^{25}$ Vgl. u. a. Hoffmann/Wille/Meinicke, Flüchtlinge und Vertriebene, S. $16 \mathrm{f}$. 
öffentlichkeitswirksam in die Umsiedlerpolitik einzubinden ${ }^{26}$. In Sachsen wurde z. B. die Leitung des Umsiedleramtes einer Vertreterin der LDP, Ruth Fabisch, überlassen, in einer „komplizierten Umarmungsstrategie“ (Michael Schwartz) bekam sie freilich „zuverlässige" Genossen als Mitarbeiter zur Seite gestellt ${ }^{27}$. Ohnehin waren die mit Aufnahme und Eingliederung der Vertriebenen verbundenen Aufgaben auf mehrere Institutionen verteilt. Ressort- und parteipolitische Konflikte können deshalb am sächsischen Beispiel im Zusammenhang untersucht werden. In die gleiche Richtung zielt die Frage, inwieweit die partielle Kompetenzübertragung an die Landtage ab Herbst 1946 den Spielraum der bürgerlichen Parteien beeinflußte: Einerseits wurde er durch Föderalisierung und Parlamentarisierung erweitert, andererseits erhielt die SED die Möglichkeit, die politische Arbeit von LDP und CDU vor dem Hintergrund einer sozialen Ausnahmesituation als überholten Vorstellungen verhaftet und unangemessen zu denunzieren. Das grundsätzliche Dilemma der bürgerlichen Parteien in der SBZ, deren aus der Weimarer Republik tradierte politische Kultur mit einem qualitativ völlig neuen und radikalen Gesellschaftsentwurf kollidierte, offenbarte sich auch in der Behandlung des Vertriebenenproblems: etwa in Forderungen nach einer kompetenten statt parteigebundenen Verwaltung oder einem rechtlich einwandfreien Lastenausgleich ${ }^{28}$.

Die nähere Untersuchung bürgerlicher Einfluß- und Gestaltungsmöglichkeiten in der Umsiedlerpolitik ist mit einer Reihe prinzipieller Fragen verbunden. Ob die LDP alternative Vorstellungen entwickeln konnte, kann erst beantwortet werden, wenn mehr Klarheit über den Spielraum der SED gegenüber der SMAD herrscht. Michael Richter hat zu Recht darauf hingewiesen, daß die fehlende Kenntnis der sowjetischen Akten nach wie vor den Eindruck begünstigt, "die SED habe in der SBZ maßgeblichen Einfluß auf die Entwicklung " besessen ${ }^{29}$. Zu klären ist darüber hinaus, ob die Vertriebenenproblematik für die LDP überhaupt Anlaß zur Entwicklung politischer Konzeptionen war, wobei allgemeinere Überlegungen etwa zur Wirtschafts- und Finanzpolitik nicht unberücksichtigt bleiben dürfen. Die Vorstellungen der Partei von Integration auf einen Nenner zu bringen, dürfte schon wegen der widersprüchlichen Haltung zur Oder-Neiße-Grenze und damit zu den Rückkehraussichten der Flüchtlinge und Vertriebenen schwierig sein. Eine Lösung des Vertriebenenproblems war für die LDP zudem lange nur im gesamtdeutschen Rahmen denkbar ${ }^{30}$.

Schließlich wird sorgfältig zu unterscheiden sein zwischen den Beschränkungen, die der LDP aus den gesellschaftspolitischen Zielvorstellungen der Sowjetunion und der SED erwuchsen, und jenen, die aus der Spezifik der Umsiedlerfrage selbst herrührten. Das Spannungsfeld zwischen politischem Anpassungsdruck, sozialen Notwendigkeiten, Ansprüchen der Parteibasis sowie bürgerlich-liberaler Tradition und Programmatik muß genauer ausgelotet werden, um in einer immanenten Perspektive der Funktionalisierung politischer Sachthemen durch die LDP nachzugehen. Am Beispiel der Arbeit der sächsischen Landtagsfraktion soll einerseits analysiert werden, wie die Artikulation der Mitgliedschaft und der Bevölkerung durch die Partei wahrgenommen und in politisches Handeln transformiert wurde, andererseits, wie die SED auf die Aktivitäten der Block-

${ }^{26}$ Schwartz, Zwischen Zusammenbruch und Stalinisierung, S. $68 \mathrm{f}$.

27 Ebenda, S. 69.

${ }^{28}$ Als Beispiel Ediger, Umsiedlerproblem, S. $9 \mathrm{ff}$.

29 Richter, Defizite, S. 164.

${ }^{30}$ Vgl. Ediger, Umsiedlerproblem, S. $4 \mathrm{ff}$. 
parteien reagierte und sie im Wechselspiel zwischen Parlament und Administration zu neutralisieren versuchte. Soweit es die Quellenlage zuläßt, soll diese Untersuchung durch lokale Fallbeispiele ergänzt werden. Die den bürgerlichen Parteien angehörenden Kommunalpolitiker waren in besonderer Weise mit dem Vertriebenennotstand konfrontiert, da SMAD und SED bestrebt waren, ihre fachliche und politische Eignung in Zweifel zu ziehen.

Spätestens nach dem Scheitern der Londoner Außenministerkonferenz im Dezember 1947 stellte die Sowjetunion ihre auf Gesamtdeutschland zielenden Konzeptionen, in denen Liberaldemokraten und der Ost-CDU wichtige Funktionen zugewiesen waren, schrittweise zugunsten der Gründung eines Separatstaates zurück ${ }^{31}$. Dementsprechend orientierte sich die SED deutlicher als bisher am Vorbild der KPdSU, artikulierte offen ihren Führungsanspruch und begann, die Gesellschaft und das politische System in ihrem Sinne umzugestalten. Der Druck auf die Führungen von LDP und CDU, die sich dem zunächst offen widersetzten, nahm beständig zu; das Vorgehen gegen frühere Sozialdemokraten in der SED signalisierte den bürgerlichen Parteien, daß das Mehrparteiensystem lediglich als Fassade aufrechterhalten werden sollte. Mit der Zulassung von DBD und NDPD (die sich im übrigen als „Umsiedlerparteien“ gerierten) im Frühjahr 1948 wurde die Rekrutierungsbasis von LDP und CDU geschmälert und das politische Kräfteverhältnis nachhaltig zugunsten der SED veränder ${ }^{32}$.

Mit den tiefgreifenden Veränderungen in der SBZ war eine Neuordnung der Umsiedlerpolitik verbunden. Die zuständigen Behörden, die Zentralverwaltung eingeschlossen, wurden aufgelöst oder institutionell zurückgestuft; die von den Landesparlamenten und -verwaltungen erlassenen Gesetze und Verordnungen einer Revision unterzogen. Gleichzeitig zog der Parteiapparat der SED die Verantwortung für Vertriebenenfragen weitgehend an sich, so daß sich die Gestaltungsmöglichkeiten der bürgerlichen Parteien endgültig auf meist karitative Begleitmaßnahmen reduzierten ${ }^{33}$.

Dennoch gibt es gute Argumente dafür, eine Untersuchung der LDP-Umsiedlerpolitik in Sachsen über diesen Einschnitt fortzuführen. So gilt es, die griffige Formel des „Einschüchterungseffekts" (Manfred Agethen), unter dem die Arbeit der bürgerlichen Parteien in der SBZ stand, mit konkreten lokalen und regionalen Beispielen zu stützen. Die Landes- und Kreisverbände der bürgerlichen Parteien und ihre parlamentarischen Vertreter waren einem anderen Druck ausgesetzt als die Berliner Zentralen ${ }^{34}$. Das nach 1948 nurmehr nachgeordnete, gleichwohl noch immer sensible Gebiet der Vertriebenenintegration verspricht Aufschluß darüber, auf welchen Politikfeldern und unter welchen Bedingungen liberaldemokratische Positionen gegen diesen Druck verteidigt werden konn$\operatorname{ten}^{35}$. Darüber hinaus kann die These überprüft werden, die LDP habe eine stärkere Anpassungsbereitschaft als die CDU gezeigt ${ }^{36}$. Schließlich ist zu analysieren, wie die veränderten politischen und institutionellen Rahmenbedingungen und der fortschreitende Einflußverlust (verbunden etwa mit der Einsicht, daß die Oder-Neiße-Linie endgültige Grenze bleiben würde) die unmittelbare Hinwendung zu den Vertriebenen verändert

31 Vgl. u. a. Agethen, CDU 1945-1953, S. 58.

32 Vgl. AdL, LV Sachsen 24886, Kurzberichte Nr. 1 und 17.

${ }^{33}$ Siehe Schwartz, Zwischen Zusammenbruch und Stalinisierung, S. 45.

${ }^{34}$ Siehe Dietze, Entscheidungsstrukturen, S. 54.

${ }^{35}$ Siehe Papke, Stand der Forschung, S. 38.

${ }^{36}$ Siehe Suckut, Wandel, S. 122. 
hat. So rückte die LDP seit 1948 schrittweise von der Forderung nach einer zonenübergreifenden Integrationspolitik $a b^{37}$. Einige Quellen legen den Schluß nahe, daß die politisch entmündigte und ihren Führungen zunehmend entfremdete Basis von Ost-CDU und LDP auch durch die gemeinsame Fürsorge für Hilfsbedürftige zusammengehalten wurde. Daß eine solche Entwicklung die von der SED bezweckte Umwandlung der bürgerlichen Parteien in bloße Transmissionsorganisationen für bestimmte soziale Schichten zumindest begünstigte (und letztlich die „offizielle“ Umsiedlerpolitik unterstützte), kann nicht übersehen werden. Desto mehr wird es darauf ankommen, Berührungspunkte und Unterschiede in der Integrationspolitik von LDP und SED, freilich auch mit vergleichendem Blick auf die CDU, detailliert herauszuarbeiten.

\subsection{Selbstbild und Erfahrungsstrukturen von Vertriebenen in Sachsen (Notker Schrammek)}

Um die vornehmlich politik- und sozialgeschichtlichen Fragestellungen der ersten drei Teilprojekte zu ergänzen, soll im vierten Teilprojekt ein Beitrag zur Alltagsgeschichte der Vertriebenen geleistet werden. Es geht darum, die Alltagsbedingungen und die Erfahrungen der Vertriebenen zu erfassen, also eine Geschichte aus der Sicht der Betroffenen zu schreiben. Die Arbeit stützt sich neben der Überlieferung in einer Reihe von Archiven auch auf Zeitzeugeninterviews.

Mit Hilfe der Methoden der Oral History ist es möglich, nach den Mentalitäten der Vertriebenen zu fragen. Im Sinne der "École des Annales" sind die Mentalitäten Gefängnisse der „longue durée“. Dieser sehr statische Begriff wurde mehrmals modifiziert ${ }^{38}$. Der französische Historiker Michel Vovelle entwickelte in seinen Arbeiten zur Französischen Revolution einen wesentlich dynamischeren Mentalitätsbegriff, mit dem er in der Lage ist, selbst den Umbruch von Mentalitäten zu beschreiben. Dieser Mentalitätsbegriff soll nun auf die Vertriebenen angewandt werden. Zum einen soll nach den Sorgen, die die Vertriebenen damals bewegten, und nach deren individueller und kollektiver Bewältigung gefragt werden. Zum anderen hatten viele Vertriebene mit dem Neuanfang in Sachsen auch große Hoffnungen verbunden. Ihre - zuweilen utopischen - Hoffnungen sollen benannt werden und es soll gefragt werden, inwieweit sie verwirklicht werden konnten. Letztlich geht es immer um die Frage, ob sich eine spezifische Vertriebenenmentalität rekonstruieren läßt. Es werden also in dieser Arbeit zwei unterschiedliche methodische Herangehensweisen miteinander verknüpft. Durch die Methoden der Oral History sollen Bereiche des Alltags erfaßt werden, die nur selten Widerspiegelung in den schriftlichen Quellen gefunden haben, denn die Akten beschränken sich im wesentlichen auf die administrative Umsetzung politischer Beschlüsse. Allerdings geben die Aussagen von Zeitzeugen in der Regel sehr subjektive Eindrücke wieder und unterliegen einem sich ändernden Erinnerungsvermögen. Daher werden die Ergebnisse der Interviews mit den herkömmlichen Archivquellen verglichen. Dies ermöglicht, die durch die Oral History gewonnenen Aussagen zu relativieren und gegebenenfalls zu korrigieren.

Da das Selbstbild der Vertriebenen entscheidend durch die Erlebnisse während der Vertreibung und der Aufnahme geprägt wurde, werden im ersten Teil der Arbeit die Sta-

37 Siehe Ediger, S. $4 \mathrm{ff}$.

${ }^{38}$ Vgl. Vovelle, La mentalité révolutionnaire, S. $12 \mathrm{f}$ sowic Ders., Die Französische Revolution, S. 151. Siehe auch Le Goff, Les Mentalités, S. 278-290. 
tionen der Vertriebenen seit ihrer Ankunft in Sachsen nachgezeichnet: die Transporte, die Lager, die Aufnahme in den Städten und Dörfern und die Wohn- und Lebensverhältnisse in den neuen Wohnorten. Untersucht werden hierbei sowohl die materiellen Lebensbedingungen als auch das geistige Umfeld, mit denen die Vertriebenen konfrontiert waren. Sodann werden in einem zweiten Schritt nachbarschaftliche Bindungen, Arbeitsverhältnisse und die Rolle gesellschaftlicher Organisationen als die Lebensbereiche beleuchtet, die für die Integration besonders wichtig waren. Hieran schließt sich eine Untersuchung der Bewahrung, Weiterentwicklung und Anpassung von Kulturgut und Traditionen an, die die Vertriebenen aus ihrer alten Heimat mit nach Sachsen brachten.

Der erste Forschungsabschnitt behandelt den Altag der Vertriebenen seit ihrer Ankunft in Sachsen. Dabei waren die Erlebnisse während der Transporte sehr unterschiedlich. Nach den vor Kriegsende Geflohenen kamen die Vertriebenen, die zunächst zu Fuß bis über die Oder-Neiße-Linie getrieben, später aber in Zügen in die verschiedenen Besatzungszonen gebracht wurden. Die von den frühen wilden Vertreibungen betroffenen Menschen erreichten Sachsen völlig geschwächt. Besonders in Grenznähe entstanden chaotische Verhältnisse. Anders war es für die Vertriebenen seit 1946. Sie empfanden die "Umsiedlung" oft als eine Befreiung aus den erniedrigenden Verhältnissen, in denen sie seit Kriegsende hatten leben müssen und knüpften daher große Hoffnungen an den Neuanfang in Deutschland.

Die Transporte wurden direkt zu einer vierzehntägigen Quarantäne in verschiedene Lager gebracht. Die dortigen Verhältnisse sind besonders gut dokumentiert, da mehrmals Inspektionen von seiten der Umsiedler-Abteilung der Landesregierung als auch der Besatzungsmacht durchgeführt wurden. Man erkennt eine äußerst unterschiedliche Ausstattung der Lager. Auch die ideologische Beeinflussung von seiten der KPD/SED setzte bereits - oder gerade - in den Lagern ein. Dort wurden Vortragsprogramme zu politischen Fragen organisiert, die von der SMAS kontrolliert wurden. Parallel dazu fanden kulturelle Veranstaltungen statt, die von Märchenstunden für Kinder über Kabarettund Varietévorstellungen bis zu Tanzveranstaltungen reichten. In den größeren Lagern waren Bibliotheken eingerichtet worden, deren Bestand streng überwacht wurde. „Aktuelle Presseerzeugnisse“, wie es in den Berichten heißt, lagen ständig aus. Da die Befunde der schriftlichen Quellen mit den Erinnerungen der Interviewpartner konfrontiert werden, kann das Bild der Lager um wesentliche Aspekte erweitert werden: Ein erstes Mal nach den Transporten zur Ruhe gekommen, begannen die Vertriebenen, sich erneut Gedanken über ihre Lebensmöglichkeiten in der Zukunft zu machen. Oft fanden sich ganze Dörfer in den Lagern wieder, so daß die Lagerinsassen sich kannten. Sie berieten dann z. B. gemeinsam über die künftigen beruflichen Schritte. Hierbei stellt sich auch immer die Frage, welchen Einfluß die staatliche Propaganda auf die persönlichen Entscheidungen der Vertriebenen hatte.

Nach dem Ende der Quarantänezeit wurden die Vertriebenen auf ihre künftigen Wohnorte verteilt. In Bezug auf die dortigen Lebensverhältnisse erweisen sich landesweit erhobene Statistiken zur Ausstattung der Wohnungen der „Umsiedler" mit Möbeln und sonstigen Einrichtungsgegenständen als besonders wertvoll. Für einige Kreise und kreisfreie Städte haben sich die Unterlagen bis auf die Ebene der einzelnen Gemeinden erhalten. Außerdem wurden, besonders wenn Beschwerden vorlagen, Inspektionsfahrten in verschiedene Ortschaften unternommen, die protokolliert wurden.

Im ersten Teil der Arbeit sollen auch die Strategien untersucht werden, die die Vertriebenen entwickelten, um den schwierigen Lebensverhältnissen zu begegnen. Da sie anders 
als viele Einheimische nicht auf Reserven oder Haushaltsgegenstände zurückgreifen konnten, waren sie neben den Ausgebombten (die aber meist noch Verwandte und Freunde hatten) die am meisten benachteiligte Bevölkerungsgruppe. Ihnen blieb nur die als erniedrigend empfundene Bettelei und die Suche nach Arbeitsplätzen, die eine bessere Versorgung mit Nahrungsmitteln versprachen, wobei Frauen auch Männerarbeiten übernahmen.

Dabei ist zu fragen, ob sich, durch die Not bedingt, neue Formen gegenseitiger Hilfe gebildet haben. Entwickelte sich ein besonderes Zusammengehörigkeitsgefühl und eine daraus resultierende gegenseitige Unterstützung unter den Vertriebenen oder überwogen die „klassischen“ Strukturen, die unabhängig von der Vertreibung bestanden, wie Familie, Pfarrgemeinde, Arbeitskollegen etc. als Bezugsgrößen? Hierbei muß natürlich auch immer das Verhältnis zu den Alteingesessenen im Auge behalten werden.

In den Wohnorten waren die Vertriebenen auch weiterhin der ideologischen Beeinflussung ausgesetzt. Die Hartnäckigkeit, mit der die Landesregierung die Aktionswoche „Neue Heimat - Neues Leben“ im Oktober 1948 gegen alle Widerstände organisierte und durchführen ließ, zeigt deutlich, daß es den Organisatoren um mehr als die Verteilung von Spenden an die "Umsiedler" ging. Trotz der ungeklärten finanziellen Situation infolge der Währungsreform vom Juni 1948 und bedeutender Widerstände großer Teile der Bürgermeister und der Umsiedler-Ausschüsse vor Ort, die sich - sei es berechtigt oder nur vorgeblich - gegen zusätzliche Verwaltungsarbeiten wehrten, wurde diese Veranstaltung realisiert. Die SED und - von ihr unterstützt und vorgeschoben - die Volkssolidarität wollten zeigen, daß sie das Heft des Handelns fest in der Hand hielten. Anhand der Berichte über die Durchführung der Aktionswoche "Neue Heimat - Neues Leben“, die jede Gemeinde einreichen mußte, sind Aufschlüsse über die Beteiligung der „Umsiedler" (und der gesamten Bevölkerung) an der Aktion möglich.

Im gleichen Zusammenhang werden zur weiteren Untersuchung der staatlichen Propaganda auch die Presseerzeugnisse, die sich mit der Lage der "Umsiedler" beschäftigten bzw. für sie bestimmt waren, herangezogen. Besonderen Stellenwert hat hier die Illustrierte „Die Neue Heimat". Ein weiteres Medium ist der Film. Die DDR-Wochenschau „Der Augenzeuge" verfolgte in ihren - allerdings seltenen ${ }^{39}$ - Berichten das Ziel, die sehr gute Organisation und die Sorge um die "Umsiedler" zu zeigen. So werden im Film die Eisenbahntransporte von lächelnden Bahnhofsvorstehern empfangen und das vorgestellte Quarantänelager ist voll von spielenden und lärmenden Kindern. Alles läßt eher an eine Ferienreise als an Vertreibung denken. Auch die beiden DEFA-Spielfilme „Freies Land“ und „Die Brücke“ von 1947 und 1948 thematisierten die „Umsiedler“-Frage.

Ebenfalls muß beachtet werden, daß in der unmittelbaren Nachkriegszeit Vertreter der verschiedenen Parteien sich um die "Umsiedler" mit eigenen Initiativen kümmerten und sie ansprachen. Durch die zunehmende Einengung der Arbeit der bürgerlichen Parteien und die Gründung der Nationalen Front verlieren sich die Unterschiede zwischen den Parteien ${ }^{40}$.

${ }^{39}$ Hier stellt sich die Frage, ob die Marginalität der Vertriebenen in der Wochenschau politisch gewollt war oder nur aufgrund der „Berlinozentrik“, die sich auch ansonsten nachweisen läßt, zustande kam. Das Wochenschau-Team um Kurt Mätzig und Marion Keller arbeitete in Berlin und in Potsdam-Babelsberg.

${ }^{40}$ Das läßt sich auch an der Zusammenstellung der Vorträge in den Lagern nachweisen. Vgl. die kulturpolitische Veranstaltungspläne vom 28. 10. 1947 und vom 7.5. 1948 in SächsHStA, LRS, MdI, 2352. 
Ferner werden die Reaktionen der Vertriebenen auf die Propaganda des Regimes beleuchtet. Es soll geklärt werden, wie sich Menschen in das politische System einfügten oder ob und in welchem Ausmaß sie sich zu verweigern suchten. Zeitgenössische Berichte über die Stimmung in den Lagern sowie in den Kreisen und Städten lassen die strittigen Punkte erkennen. Immer wieder tauchte als die zentrale Frage die Akzeptanz der Oder-Neiße-Linie auf. Ständig wurde berichtet, daß die Vertriebenen noch immer den Wunsch gezeigt hätten, in ihre Heimat zurückzukehren.

Nach der Schilderung des Alltags soll der zweite Teil der Arbeit besonders die Lebensbereiche beleuchten, die als Brücken zum Einstieg in das gesellschaftliche Leben in Sachsen betrachtet werden können. Hierbei wird das Verhältnis zu den Nachbarn, die Kontakte am Arbeitsplatz sowie die Rolle gesellschaftlicher Organisationen und der Kirchen $\mathrm{zu}$ untersuchen sein.

Zuerst sollen in diesem Zusammenhang die nachbarschaftlichen Bindungen untersucht werden. Die Untermietverhältnisse, die die Vertriebenen in den meisten Fällen eingehen mußten, gestalteten sich nicht einfach. Besonders die gemeinsame Benutzung von Küche, Bad und Toiletten sowie die gemeinsamen Rechnungen für Strom und Gas bargen viel Konfliktstoff ${ }^{41}$. Des weiteren sind die Arbeitsverhältnisse zu beachten, die neben der Wohnungsbereitstellung der wohl wichtigste Integrationspunkt waren, selbst wenn es immer wieder vorkam, daß „Flüchtlinge ${ }^{\text {‘ } 42}$ in den Arbeitsgruppen an den Rand gedrängt wurden. Für die Kinder kann man die gleiche Frage in Bezug auf die Schule stellen. Hier kommt noch hinzu, daß viele Kinder „überaltert“ waren, da ihnen seit Kriegsende der Schulbesuch in den Vertreibungsgebieten verwehrt wurde. Andere Schüler hatten Schwierigkeiten, da sie, wie etwa Ungarndeutsche, zuvor keine deutschsprachige Schule besucht hatten. Schließlich werden in diesem Zusammenhang auch gesellschaftliche Organisationen wie die FDJ und die Parteien als besonders wichtige Integrationsfelder untersucht. Die Motivationen für die Mitgliedschaft in diesen gesellschaftlichen Organisationen sollen beleuchtet werden. Zu bedenken ist ebenfalls, daß in diesen Organisationen zum Teil Formen des gesellschaftlichen Umgangs herrschten, die denen der nationalsozialistischen Organisationen vergleichbar waren: Gemeinsame Ausflüge, Lager, Singgruppen etc. konnten daher als Anküpfungspunkte empfunden werden.

Viele Vertriebene suchten in den Kirchen ihrer neuen Heimat Kontakt. Hier ist etwa zu fragen, wie die Kirchgemeinden die Neuankömmlinge aufnahmen, die oft aus anderen kirchlichen Traditionen und Frömmigkeitsformen kamen. Vertriebene Pfarrer mußten in die Sächsische Landeskirche bzw. das katholische Bistum Meißen integriert und mit einem Minimum an Arbeitsmitteln ausgestattet werden. Erschwerend wirkte zudem noch, daß die evangelische Kirche sich in einer Phase der Aufarbeitung der eigenen Vergangenheit befand, die zusätzliche Spannungen entstehen ließ.

Katholische Gemeinden entstanden in Sachsen in vielen Städten erst neu oder vergrößerten sich um ein Vielfaches durch den Zustrom der Vertriebenen. In gewisser Weise deckte sich hier das Selbstverständnis als Katholik mit dem als Vertriebener. Die Notlage - es fehlten im allgemeinen die katholischen Kirchengebäude - und die gemeinsame Er-

${ }^{41}$ In den Protokollen des Umsiedlerausschusses der Stadt Werdau erkennt man, daß die meisten Beschwerden aufgrund von Streitigkeiten mit dem Hauptmieter vorgetragen werden. In: Stadtarchiv Werdau, Soz 1801/1.

${ }^{42}$ Das Wort „Flüchtling“ in der sächsischen Aussprache des „ü“ als ein „i“ hat sich in das Gedächtnis der Vertriebenen als ein Schimpfwort eingegraben. 
fahrung, durch den Staat an den Rand gedrängt zu werden, gaben erste Anstöße zu einer ökumenischen Zusammenarbeit zwischen den Kirchen.

Die drei genannten Integrationsfelder Nachbarschaft, Arbeit und gesellschaftliche Gruppen wurden in den verschiedenen Ansiedlungsgebieten unterschiedlich erfahren. Deshalb soll untersucht werden, ob die Anonymität der Großstadt einen Vorteil gegenüber den eher verschlossenen Dorfgemeinschaften darstellte, ob die stark industrialisierten Gebiete Westsachsens mit ihren vielen Arbeitsmöglichkeiten bessere Bedingungen für die Integration boten als die landwirtschaftlichen Gebiete Ostsachsens, wo die Arbeit nur saisonweise anfiel und in Naturalien vergütet wurde und welche Rolle die Grenznähe spielte.

In einem dritten Teil der Arbeit sollen die von den Vertriebenen bewußt bewahrten landsmannschaftlichen Besonderheiten, Traditionen und Bräuche Gegenstand der Untersuchung sein. Sie haben trotz aller Unterdrückung durch materielle Zwänge und staatliche Propaganda weitergelebt, reichten von Kleidungs- und Eßgewohnheiten bis hin zur mundartgefärbten Sprache. Auch die Kirchen entwickelten eine spezielle Seelsorge für Vertriebene mit eigenen Gottesdiensten und besonderen Predigten.

Als ein Indikator für den Erfolg der Integration kann das Heiratsverhalten der Vertriebenen angesehen werden. Dazu soll herausgearbeitet werden, bis zu welchem Zeitpunkt Vertriebene bevorzugt unter sich geheiratet haben bzw. seit wann die Ehe mit Einheimischen zur Normalität wurde. Weiterhin soll untersucht werden, ob der Anteil unehelicher Geburten unter den Vertriebenen vom allgemeinen Durchschnitt der Gesellschaft abweicht, um hieraus Rückschlüsse auf die gesellschaftliche Akzeptanz "gemischter" Ehen zwischen Alteingesessenen und Vertriebenen zu ziehen. Abschließend sind durch eine Analyse der sekundären Migration der Vertriebenen aus Sachsen nach den westlichen Besatzungszonen bzw. in die Bundesrepublik Rückschlüsse auf die Tiefe der Verwurzelung der Vertriebenen in Sachsen möglich. 